Pacific Journal of Mathematics

ENDS OF FUNDAMENTAL GROUPS IN SHAPE AND PROPER
HOMOTOPY 


\title{
ENDS OF FUNDAMENTAL GROUPS IN SHAPE AND PROPER HOMOTOPY
}

\author{
Michael L. Mihalik
}

The number of topological ends of the universal cover of a finite complex, $K$, is either $0,1,2$, or $\infty$ and only depends upon the fundamental group of $K$. Call this number $e(K)$. We wish to define numbers $e(X)$ for compact metric spaces analogous to $e(K)$. To accomplish this we extend the theory of ends for finitely generated groups to certain inverse sequences of finitely generated groups and their inverse limits. Classifications for these inverse sequences and their inverse limits analogous to those for finitely generated groups are derived. Whenever the fundamental pro-group of a compact metric space, $X$, satisfies certain properties, we obtain a shape invariant number $e(X)$ (either $0,1,2$ or $\infty$ ) and analyze what $e(X)$ describes geometrically.

1. Introduction. The number of ends of a topological space was introduced by Freudenthal in [8]. Let $X$ be a locally compact separable metric space. Let $\left\{C_{i}\right\}_{i=1}^{\infty}$ be a collection of compact subsets of $X$ such that $C_{i} \subset \operatorname{int}\left(C_{i+1}\right)$ (the interior of $\left.C_{i+1}\right)$ and $\bigcup_{i=1}^{\infty} C_{i}=X$. The cardinality of $\lim \left\{\pi_{0}\left(X-C_{i}\right)\right\}$ (where the bonds are induced by inclusions) is the number of ends of $X$. This number is independent of the choice of the $C_{i}$.

In [10] Hopf proves:

(i) The universal cover, $\widetilde{K}$, of a compact polyhedron has either $0,1,2$, or $\infty$-ends.

Call this number $e(K)$.

(ii) If $K_{1}$ and $K_{2}$ are compact polyhedra and $\pi_{1}\left(K_{1}\right)=\pi_{1}\left(K_{2}\right)$ then $e\left(K_{1}\right)=e\left(K_{2}\right)$.

This paper is motivated by the desire to extend Hopf's theorem to compacta i.e., if $X$ is a compact connected metric space we would like numbers $e(X)$ analogous to Hopf's $e(K)$. In $\S 5$ we accomplish this for a large class of compacta, with $e(X)$ a shape invariant of $X$. Geometrically $e(X)$ is counting the number of ends of the universal covers of certain compact polyhedra associated with $X$. We also derive sufficient conditions to obtain a space, $\bar{X}$, associate with $X$ (analogous to the universal cover of $K$ ) so that the number of ends of $\bar{X}$ is $e(X)$. This $\bar{X}$ will reduce to the universal cover whenever $X$ is $L C^{\circ}$ and semi-locally 1-connected. With mild restrictions we show if $X$ and $Y$ are pointed homotopy equivalent then $\bar{X}$ and $\bar{Y}$ are pointed proper homotopy equivalent.

Let $Y$ be a locally compact separable metric space with one 
end, and $\left\{C_{i}\right\}$ a collection of compact subsets such that $C_{i} \subset \operatorname{int}\left(C_{i+1}\right)$ and $\mathrm{U}_{i=1}^{\infty} C_{i}=Y$. If the inverse sequence $\left\{\pi_{1}\left(Y-C_{i}\right)\right\}$ (with bonding homomorphisms induced by inclusions and proper attention to the base points) satisfies conditions described in $\S 3$ then a theory for the number of ends of the fundamental group of the end of $Y$ can be derived. It is a well-known conjecture that these conditions are satisfied whenever $Y$ is the universal cover of a finite complex. We do not explore this avenue in this paper although the basic tools are implicitly evident. This is one reason for the words "and proper homotopy" in the title of the paper.

The results of Freudenthal [8] and Hopf [10] led to the following group theory:

If $G$ is a finitely generated group there is a number, $e(G)$, called the number of ends of $G$ such that:

(i) $e(G)$ is either $0,1,2$, or $\infty$.

(ii) If $K$ is a compact polyhedron and $\pi_{1}(K) \cong G$ then $e(G)=$ $e(K)$.

(iii) $e(G)=0$ if and only if $G$ is finite.

(iv) $e(G)=2$ if and only if $G$ has an infinite cyclic subgroup finite index.

(v) (Stallings) $e(G)=\infty$ if and only if $G$ is an amalgamated free product or HNN extension of a certain type (see $\S 3$ ).

(vi) $e(G)=1$ otherwise.

Our methods naturally lead us to generalize these results about ends of groups in two ways: to suitable inverse sequences of groups (objects in the category of pro-groups) in $\S 3$, and to suitable topological groups in $\S 4$. [All this is carefully explained in those sections, but a reader familiar with the shape theory of compacta will not be surprised that we approach the desired geometrical theorems about $e(X)$ through inverse sequences of groups and/or their topological inverse limits.] The geometrical theorems, described above, are in $\S 5$.

Finally, there are several unexpected features, two of which are:

1. The compacta for which $e(X)$ is defined are pointed 1-movable (the fundamental pro-group is Mittag-Leffler (M-L)), and the bonding homomorphisms (of the fundamental pro-group) have finite kernel. Although the pointed 1-movable condition is a frequently used concept the finite kernel condition has not previously been used. We call this condition Mittag-Leffler finite (MLF).

2. 2-ended finitely generated groups can be classified as those with a normal infinite cyclic subgroup of finite index. In $\S 4$ we describe a 2-ended topological group with no normal infinite cyclic subgroup of any sort. 
3. The condition MLF is not an ad hoc condition. The class of compacta with MLF fundamental pro-groups appears to be the natural class for a theory of ends.

The content of this paper is taken from the author's dissertation written at the State University of New York at Binghamton under the direction of Ross Geoghegan.

2. Ends and homomorphisms with finite kernel. All groups considered in $\S 2$ will be finitely generated.

Let $G$ be a finitely generated group with specified generators $\left\langle g_{1}, \cdots, g_{n}\right\rangle$. Construct a 1-complex $L\left(g_{1}, \cdots, g_{n}\right)$ with one vertex for each element of $G$ and one edge joining verticies $a$ and $b$ if $a g_{i}=b$ for some $i \in\{1,2, \cdots, n\}$. The number of ends of $L\left(g_{1}, \cdots, g_{n}\right.$ ) (see $\S 1$ for the meaning of this) does not depend on the choice of $g_{1}, \cdots, g_{n}[10]$.

Definition. The number of ends of $G$ is the number of ends of $L\left(g_{1}, \cdots, g_{n}\right)$ in the topological sense.

Proofs of the following two theorems can be found in Stallings [14] (p. 54 and p. 38 respectively).

THEOREM 2.1. A finitely generated group has either 0, 1, 2 or $\infty$-ends.

TheOREM 2.2. A finitely generated group has 2-ends if and only if it has an infinite cyclic subgroup of finite index.

As a companion to these two theorems we state the trivial:

Proposition 2.3. A finitely generated group has 0-ends if and only if it is finite.

The main result of this section is the following:

Proposition 2.4. If $G$ and $H$ are finitely generated groups and $f: G \rightarrow H$ is an epimorphism with finite kernel then $G$ and $H$ have the same number of ends.

The proof will be done as a sequence of lemmas.

Lemma 2.5. If $f$ is as in 2.4 then $G$ is 0-ended if and only if $H$ is 0-ended.

This is trivial. 
Lemma 2.6. Let $G$ be 2-ended and let $f: G \rightarrow H$ be an epimorphism whose image $H$, is infinite then $H$ is 2-ended and $f$ has finite kernel.

Proof. Let $a$ be a generator of $Z_{a}$, an infinite cyclic subgroup of finite index in $G$. Let $\left[x_{1}\right], \cdots,\left[x_{n}\right]$ be the right cosets of $G / Z_{a}$. $\left(p \in\left[x_{i}\right]\right.$ if and only if $p=a^{k} x_{i}$ for some integer $k$.) $f(a)$ has infinite order in $H$, since if not $H$ would be finite, contrary to assumption. It suffices to observe that if $b$ and $c$ are in the same right coset of $G / Z_{a}$ then $f(b)$ and $f(c)$ are in the same right coset of $H / Z_{f(a)}$ i.e., there are at most $n$ right cosets in $H / Z_{f(a)}$.

To see $f$ has finite kernel, one need only observe that $f$ is $1-1$ on the elements of a right coset [xi] . I.e., for $k \neq m f\left(a^{k} x_{i}\right)=$ $(f(a))^{k} f\left(x_{i}\right) \neq(f(a))^{m} f\left(x_{i}\right)=f\left(a^{m} x_{i}\right)$ since $f(a)$ has infinite order in $H$.

Lemma 2.7. Let $H$ be 2-ended and let $f: G \rightarrow H$ be an epimorphism with finite kernel then $G$ is 2-ended.

Proof. Say $x$ generates $Z_{x}$ an infinite cyclic subgroup of finite index in $H$. If $y \in f^{-1}(x)$ then $y$ has infinite order in $G$ and $f$ maps each right coset of $G / Z_{y}$ bijectively to one of $G / Z_{x}$. Thus if $G / Z_{y}$ were infinite $f$ would not be finite-to-one i.e., $f$ would have infinite kernel, contrary to assumption.

LEMмA 2.8. If $f: G \rightarrow H$ is an epimorphism with finite kernel then $G$ is $\infty$-ended if and only if $H$ is $\infty$-ended.

Proof of "only if". Let $\left\{g_{1}, \cdots, g_{t}\right\}$ generate $G$. Then $\left\{f\left(g_{1}\right), \cdots, f\left(g_{t}\right)\right\}$ generates $H$. Let $L_{1}=L\left(g_{1}, \cdots, g_{t}\right)$, and $L_{2}=$ $L\left(f\left(g_{1}\right), \cdots, f\left(g_{t}\right)\right) f$ induces $f_{1}: L_{1} \rightarrow L_{2}$ which is a covering projection with fiber of the same cardinality as $\operatorname{ker}(f)$. Hence $f_{1}$ is proper. Choose $C$ a compact subset of $L_{1}$ such that $L_{1}-C$ has $n$-infinite components, where $n>|\operatorname{ker}(f)| . \quad f_{1}^{-1} f_{1}(C)$ is a compact set containing $C$ and $L_{1}-f_{1}^{-1} f_{1}(C)$ has $m$-infinite components with $m \geqq n$. The infinite components of $L_{1}-f_{1}^{-1} f_{1}(C)$ cover the infinite components of $L_{2}-f_{1}(C)$. But at most $|\operatorname{ker}(f)|$ infinite components of $L_{1}-f_{1}^{-1} f_{1}(C)$ can cover any particular infinite component of $L_{2}$ $f_{1}(C)$. Hence $L_{2}-f_{1}(C)$ must have at least two infinite components, and $L_{2}$ has at least two ends. By Lemma 2.7, $L_{2}$ is not 2-ended, and therefore $L_{2}$ is $\infty$-ended.

Proof of "if". Choose $D$ a compact subset of $L_{2}$ such that $L_{2}-D$ has three or more infinite components. If $D_{1}, D_{2}$ and $D_{3}$ are such components, then $f_{1}^{-1}\left(D_{i}\right)$ for $i \in\{1,2,3\}$ contains an infinite 
component of $L_{1}-f_{1}^{-1}(D)$ i.e., $L_{1}$ has at least three ends and therefore $G$ is $\infty$-ended.

Lemma 2.5 is the 0-ended case of Proposition 2.4, Lemmas 2.6 and 2.7 the 2-ended case and Lemma 2.8 the $\infty$-ended case. Thus the only remaining case, the 1-ended case also holds, completing the proof of Proposition 2.4.

3. Inverse sequences of groups. Let $G_{1} \stackrel{f_{1}}{\longleftarrow} G_{2} \stackrel{f_{2}}{\longleftarrow} \ldots$ be an inverse sequence of groups and homomorphisms. The sequence is Mittag-Leffler (M-L) [9] if given $n>0$, there is $m(n) \geqq n$ such that $\operatorname{im}\left(G_{m(n)+k} \rightarrow G_{n}\right)=\operatorname{im}\left(G_{m(n)} \rightarrow G_{n}\right)$ for all $k \geqq 0$. If every bond is onto or each $G_{n}$ is finite, then clearly $\left\{G_{n}, f_{n}\right\}$ is M-L. The sequences $\left\{G_{n}, f_{n}\right\}$ and $\left\{H_{n}, k_{n}\right\}$ are pro-isomorphic if there exists cofinal subsequences $\left\{G_{m(j)}\right\}_{j=1}^{\infty}$ and $\left\{H_{m(j)}\right\}_{j=1}^{\infty}$ and homomorphisms $p_{j}$ and $q_{j}$ making diagram (A) commute for all $j$.

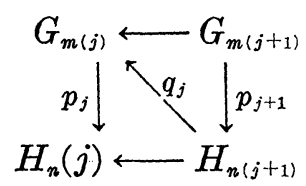

where the horizontal homomorphisms are compositions of bonds. (This and related notions were introduced in [2].) Clearly pro-isomorphism is an equivalence relation. It is easy to see that any M-L system is pro-isomorphic to one with epimorphic bonds. We are interested in $\mathrm{M}-\mathrm{L}$ inverse sequences of finitely generated groups in which the bonding homomorphisms have finite kernels. We call such sequences Mittag-Leffler-finite (M-L-F). By Proposition 2.4 , if $\left\{G_{i}, f_{i}\right\}$ is an M-L-F sequence of finitely generated groups and each $f_{i}$ is an epimorphism then the $G_{i}$ all have the same number of ends.

Proposition 3.1. If $\left\{H_{i}, k_{i}\right\}$ and $\left\{G_{i}, f_{i}\right\}$ are pro-isomorphic M-L-F sequences with epimorphic bonds then all groups $G_{i}, i \epsilon$ $\{1,2, \cdots\}$ and $H_{i}, i \in\{1,2, \cdots\}$ have the same number of ends.

Proof. By Proposition 2.4 it suffices to show the number of ends of $G_{i}$ equals the number of ends of $H_{j}$ for some $i$ and $j$. Assume $p_{i}$ and $q_{i}$ are homomorphisms making diagram (B) commute for all $i$.

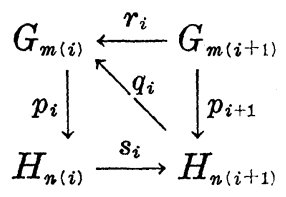


$r_{i}$ and $s_{i}$ are the appropriate compositions of bonds, and thus are epimorphisms with finite $\operatorname{kernels.} \operatorname{ker}\left(p_{i}\right) \subset \operatorname{ker}\left(q_{i-1} p_{i}\right)=\operatorname{ker}\left(r_{i-1}\right)$, and $\operatorname{ker}\left(p_{i}\right)$ is finite. $\quad p_{i}$ is an epimorphism since $p_{i} \circ q_{i}=s_{i}$ is. By Proposition $2.4 G_{m(i)}$ and $H_{n(i)}$ have the same number of ends.

Definition. The number of ends of the M-L-F sequence $\left\{G_{i}, f_{i}\right\}$ of finitely generated groups is the number of ends of the $G_{i}^{\prime}$ where $\left\{G_{i}^{\prime}, f_{i}^{\prime}\right\}$ is any M-L-F sequence pro-isomorphic to $\left\{G_{i}, f_{i}\right\}$ and each $f_{i}^{\prime}$ is an epimorphism. This definition is unambiguous by Proposition 3.1 .

Proposition 3.2. If $\left\{G_{i}, f_{i}\right\}$ is M-L-F and each $f_{i}$ is an epimorphism then $G_{1}$ finite implies $\left\{G_{i}, f_{i}\right\}$ is 0 -ended, and $G_{1}$ having an infinite cyclic subgroup of finite index implies $\left\{G_{i}, f_{i}\right\}$ is 2ended.

Proof. The first part is trivial and the second follows from Theorem 2.2.

Let $G$ be a group, $A$ and $B$ subgroups of $G$ and let $\theta: A \rightarrow B$ be an isomorphism. The HNN extension of $G$ relative to $A, B$ and $\theta$ is the group $G \varsigma_{A} \theta=\left\langle G, x \mid x^{-1} a x=\theta(a), a \in A\right\rangle$, i.e., the generators and relations of (some presentation of) $G$, together with an additional generator, $x$, and additional relations $x^{-1} a x=\theta(a)$. (See [12] p. 179.) An alternative description of $\mathrm{HNN}$ extensions can be found in [14] (3.A.5.5) in terms of pre-groups. We avoid pre-groups in this paper.

By [14] (p. 57) the finitely generated group $G$ has an infinite number of ends if and only if either

(1) $G=G_{1} *_{F} G_{2}$, a free product with finite amalgamated subgroup $F$, properly contained in both factors, and of index $>2$ in at least one factor, or

(2) $G=H \hookrightarrow_{F} \theta$ an HNN extension, where $F$ is a finite subgroup properly embedded in $H$. Thus,

Proposition 3.3. If $\left\{G_{i}, f_{i}\right\}$ is M-L-F, each $f_{i}$ is an epimorphism and $G_{1}$ is a free product with finite amalgamated subgroup as in (1) or an HNN extension as in (2) then $\left\{G_{i}, f_{1}\right\}$ is $\infty$-ended.

Proposition 3.4. If $\left\{G_{i}, f_{i}\right\}$ is 0-ended and each $f_{i}$ is an epimorphism then all $G_{i}$ are finite.

This is trivial. 
Proposition 3.5. If $\left\{G_{i}, f_{i}\right\}$ is 2-ended and each $f_{2}$ is an epimorphism then there is $x_{n} \in G_{n}$ for each $n$ such that $f_{n}\left(x_{n+1}\right)=x_{n}$ and $x_{n}$ generates an infinite cyclic subgroup of finite index in $G_{n}$.

Proof. In a group with infinite cyclic subgroup of finite index any element of infinite order generates an infinite cyclic subgroup of finite index. By Theorem 2.2, each $G_{n}$ has an infinite cyclic subgroup of finite index thus if $x_{1}$ has infinite order in $G_{1}$ and $x_{n}$ is selected such that $f_{n-1}\left(x_{n}\right)=x_{n-1}$ for each $n \geqq 2$ then $x_{n}$ has infinite order in $G_{n}$ and generates in infinite cyclic subgroup of finite index in $G_{n}$.

Finitely generated groups with 2-ends have normal infinite cyclic subgroups of finite index. The following example shows that in a 2-ended M-L-F sequence with epimorphic bounds it may not be possible to choose compatible normal infinite cyclic subgroups i.e., there need not exist $x_{n} \in G_{n}$ such that $x_{n}$ generates a normal infinite cyclic subgroup in $G_{n}$ and $f_{n}\left(x_{n+1}\right)=x_{n}$ for all $n$.

EXAMPlE 3.6. Denote the permutation group on $n$ symbols as $S_{n}$. The following are easily checked in $S_{2^{n}}$.
(i) $\left[1,2, \cdots, 2^{n}\right][a, a+1]=\left[1,2, \cdots, a, a+2, \cdots, 2^{n}\right]=[a+1$, $a+2]\left[1,2, \cdots, 2^{n}\right]$ for $a \in\left\{1,2, \cdots, 2^{n}-2\right\}$
(ii) $\left[1,2, \cdots, 2^{n}\right]\left[2^{n}, 1\right]=\left[2,3, \cdots, 2^{n}\right]=[1,2]\left[1,2, \cdots, 2^{n}\right]$
(iii) $\left[1,2, \cdots, 2^{n}\right]\left[2^{n}-1,2^{n}\right]=\left[1,2, \cdots, 2^{n}-1\right]=\left[2^{n}, 1\right]$ $\left[1,2, \cdots, 2^{n}\right]$
(iv) $\left[1,2, \cdots, 2^{n}\right]^{2}[a, a+1]=[a+2, a+3]\left[1,2, \cdots, 2^{n}\right]^{2}$ for $a \in$ $\left\{1,2, \cdots, 2^{n-3}\right\}$
(v) $\left[1,2, \cdots, 2^{n}\right]^{2}\left[2^{n}-1,2^{n}\right]=[1,2]\left[1,2, \cdots, 2^{n}\right]^{2}$.

Lemma 3.6.1. If $x$ generates $Z_{x}$, a normal infinite cyclic subgroup of $G$, then $g x g^{-1}=x$ or $x^{-1}$ for any $g \in G$.

Proof. Say $g x g^{-1}=x^{n}$ and $g^{-1} x g=x^{m}$ for some integers $m$ and $n$. Then $x=g^{-1} g x g^{-1} g=g^{-1} x^{n} g=\left(x^{n}\right)^{m}=x^{n m}$. Thus $n \cdot m=1$ and $n=m=1$ or $n=m=-1$.

Now we define our M-L-F sequence. Let $G_{1}$ be the infinite cyclic group with generator $x_{1}$. For $n \geqq 2, G_{n}=\left\langle x_{n} ; a_{1, n} ; \cdots\right.$; $a_{2^{n-1} n} \mid x_{n} a_{i, n}=a_{i+1, n} x_{n}$ for $i \in\left\{1, \cdots, 2^{n-1} 1\right\} ; x_{n} a_{2^{n-1, n}}=a_{1, n} x_{n} ; a_{i, n}^{2}=1$ for all $i ; a_{i, n} a_{j, n}=a_{j, n} a_{i, n}$ for all $i$ and $\left.j\right\rangle$. Define $f_{1}: G_{2} \rightarrow G_{1}$ by $x_{2} \rightarrow x_{1}$ and $a_{i, 2} \rightarrow 1$ for $i=1$ or 2 . For $n \geqq 2$, define $f_{n}: G_{n+1} \rightarrow G_{n}$ by $x_{n+1} \rightarrow x_{n}, a_{i, n+1} \rightarrow a_{i, n}$ for $i \in\left\{1,2, \cdots, 2^{n-1}\right\}$ and $a_{2^{n-1+i, n+1}} \rightarrow a_{i, n}$ for $i \in\left\{1,2, \cdots, 2^{n-1}\right\}$.

(vi) Since $f_{1} \circ \cdots \circ f_{n-1}\left(x_{n}\right)=x_{1}, x_{n}$ has infinite order.

(vii) The relations of $G_{n}$ easily imply any word in $G_{n}$ can be 
written as $a_{1, n}^{t(1)} \cdots a_{2_{n-1, n}}^{t\left(2^{n-1}\right)} x_{n}^{k}$ where $t(i) \in\{0,1\}$ and $k$ is an integer.

(viii) The words of $G_{n}$ that are mapped to $x_{1}^{k}$ are exactly those of the form in (vii).

From (vii) the infinite cyclic subgroup of $G_{n}$ generated by $x_{n}$ has finite index in $G_{n}$, and $G_{n}$ is 2-ended $f_{n}$ has finite kernel by Lemma 2.6 and is an epimorphism by definition. Thus $\left\{G_{i}, f_{i}\right\}$ is M-L- $F$. For $n \geqq 2$, define $g_{n}: G_{n} \rightarrow S_{2^{n}}$ by $x_{n} \rightarrow\left[1,2, \cdots, 2^{n}\right]^{2}$ and $a_{i, n} \rightarrow[2 i-1,2 i]$ for $i \in\left\{1,2, \cdots, 2^{n-1}\right\}$. By (iv) and (v) $g_{n}$ is a homomorphism. In particular,

(ix) $a_{i, n} \neq a_{j, n}$ for $i \neq j$.

LemMA 3.6.2. If $G_{n}$ has a normal infinite cyclic subgroup, then a generator of it must have the form $a_{1, n}^{t(n)} \cdots a_{2 n-1, n}^{t\left(2_{n}^{n-1}\right)} x_{n}^{K 2^{n-1}}$ for $K$ a nonzero integer and $t_{i} \in\{0,1\}$.

Proof. $\quad x_{n}^{2^{n-1}} a_{i, n}=a_{i, n} x_{n}^{2^{n-1}}$ for all $i$, thus $x_{n}^{-2^{n-1}} a_{i, n}=a_{i, n} x_{n}^{-2^{n-1}}$ and for any integer $K, a_{i, n} x_{n}^{K^{2 n-1}}=x_{n}^{K 2^{n-2}} a_{i, n}$ for all $i$. If $L \in\{1,2, \cdots$, $\left.2^{n-1}-1\right\}$ then $x_{n}^{L} a_{1, n}=a_{1+L, n} x_{n}^{L}$. Thus if $W=a_{1, n}^{t(1)} \cdots a_{2^{2}-1, n}^{t\left(2^{n-1}\right)} x_{n}^{K 2^{n-1+L}}$ for $t(i) \in\{0,1\}, L \in\left\{1,2, \cdots, 2^{n-1}-1\right\}$ and $K$ is an integer, it suffices to show $a_{1, n} W a_{1, n} \neq W$ or $W^{-1}$. By Lemma 3.6.1 $a_{1, n} W a_{1, n}$ is mapped to $x_{1}^{K 2^{2-1+L}}$ under bonding homomorphisms, and $W^{-1}=$ $x_{n}^{-K 2^{n-1}-L} a_{1, n}^{t(1)} \cdots a_{2^{n-1, n}}^{t\left(2^{n-1}\right)}$ is mapped to $x_{1}^{-K 2^{n-1}-L}$ i.e., $a_{1, n} W a_{1, n} \neq W^{-1}$. $a_{1, n} W a_{1, n}=a_{1, n} a_{1+L, n} W \neq W$ by (ix).

Assume $b_{n}$ generates a normal infinite cyclic subgroup in $G_{n}$, and $f_{n}\left(b_{n+1}\right)=b_{n}$ for all $n$. Say $b_{1}=x_{1}^{m}, m \neq 0$, then by (viii) $b_{p}=$ $a_{1, p}^{t(1)} \cdots a_{2^{p-1} p}^{t\left(2^{p-1}\right)} x_{p}^{m}$ for $t(i) \in\{0,1\}$. But for $2^{p-1}>|m|, m$ is not a multiple of $2^{p-1}$ and Lemma 3.6.2 implies $b_{p}$ does not generate a normal infinite cyclic subgroup in $G_{p}$.

An alternative characterization of 2-ended groups is as follows: $G$ is 2-ended if and only if $G$ has a finite normal subgroup $F$ such that $G / F$ is infinite cyclic or is the infinite dihedral group $Z_{2} * Z_{2}$ [15] (p. 38). This motivates the following:

Lemma 3.7. If $\left\{G_{i}, f_{i}\right\}$ is a 2-ended M-L-F sequence, and each $f_{i}$ is an epimorphism then there is a compatible sequence of finite normal subgroups $F_{i} \subset G_{i}$ (compatible in the sense $f_{n}^{-1}\left(F_{n}\right)=F_{n+1}$ ) such that $G_{i} / F_{i}$ is infinite cyclic for all $i$, or $Z_{2} * Z_{2}$ for all $i$.

Proof. Since $G_{1}$ is 2-ended there is $F_{1}$, a normal finite subgroup of $G_{1}$, such that $G_{1} / F_{1}$ is the integers, $Z$, or $Z_{2} * Z_{2}$. Let $f_{0}: G_{1} \rightarrow Z$ or $Z_{2} * Z_{2}$ be the quotient homomorphism. $f_{0} \circ f_{1}: G_{2} \rightarrow Z$ or $Z_{2} * Z_{2}$ is an epimorphism with kernel $f_{1}^{-1}\left(F_{2}\right)$. Thus $G_{2} / f_{1}^{-1}\left(F_{1}\right)=Z$ or $Z_{2} * Z_{2}$. Inductively define $F_{n}=f_{n-1}^{-1}\left(F_{n-1}\right)$, for $n \geqq 2$. The kernel of $f_{0} \circ \cdots \circ f_{n-1}$ is $F_{n}$ and since each $f_{i}$ has finite kernel, $F_{n}$ is finite 
and normal and $G_{n} / F_{n}=Z$ or $Z_{2} * Z_{2}$ for all $n$.

It remains to characterize $\infty$-ended M-L-F sequences.

A bipolar structure on a group $G$, as defined in Stallings [14] is a partition of $G$ into six disjoint sets, termed $F, S, E E, E E^{*}$, $E^{*} E, E^{*} E^{*}$ satisfying the axioms below. We let $X, Y, Z$ be symbols standing for the letters $E$ or $E^{*}$, and if $X=E$ or $E^{*}$ then $X^{*}=E^{*}$ or $E$ respectively.

Axioms.

1. $F$ is a finite subgroup of $G$.

2. $F \cup S$ is a subgroup of $G$ in which $F$ has index 1 or 2 .

3. If $f \in F, g \in X Y$, then $f g \in X Y$.

4. If $s \in S, g \in X Y$ then $g s \in X Y^{*}$.

5. If $g \in X Y$, then $g^{-1} \in Y X$.

6. If $g \in X Y, h \in Y^{*} Z$ then $g h \in X Z$.

7. If $g \in G$, there exists a minimal integer, $N(g)$ such that whenever $g_{1}, \cdots, g_{n} \in G$ and there exists $X_{0}, \cdots, X_{n}$ such that $g_{i} \in$ $X_{i-1}^{*} X_{i}$ and $g=g_{1} \cdots g_{n}$ then $n \leqq N(g)$.

8. $E E^{*} \neq \varnothing$.

If $G$ is a group with bipolar structure then $P \in G$ is irreducible if Axiom 7 holds with $N(P)=1$. Thus $P$ is irreducible if $P \in F \cup S$ or if $P$ cannot be written as $g \cdot h$ with $g \in X Y$ and $h \in Y^{*} Z$. If $G$ has a bipolar structure, then $\left(x_{1}, x_{2}, \cdots, x_{n}\right)$ is a reduced word if each $x_{i}$ is irreducible, $x_{1} \notin F \cup S$, and $x_{i} \in X Y$ implies $x_{i+1} \in Y^{*} Z$ for $i \in\{1,2, \cdots, n-1\}$, or $x_{1} \in F \cup S$ and $n=1$.

LEMMA 3.8. If $f: H \rightarrow G$ is an epimorphism with finite kernel and $G$ has bipolar structure with partition $F, S, E E, E E^{*}, E^{*} E$, $E^{*} E^{*}$, then $H$ has bipolar structure with partition $f^{-1}(F), f^{-1}(S)$, $f^{-1}(E E), f^{-1}\left(E E^{*}\right), f^{-1}\left(E^{*} E\right), f^{-1}\left(E^{*} E^{*}\right)$.

The proof is immediate since (in Axiom 7) $N(h)$ is bounded above by $N(f(h))$. for any $h \in H$.

Leмma 3.9. If $f: H \rightarrow G$ is an epimorphism with finite kernel and $G$ has bipolar structure, then $N(h)=N(f(h))$ for any $h \in H$.

Proof, If $\left(x_{1}, \cdots, x_{n}\right)$ is reduced and $h=x_{1} \cdots x_{n}$ then $N(h)=n$ by [14] (p. 32). To see $N(f(h))=n$ it remains to show $\left(f\left(x_{1}\right), \cdots\right.$, $\left.f\left(x_{n}\right)\right)$ is reduced. Since $H$ obtains its bipolar structure from $f^{-1}$, we need only show: If $x \in H-f^{-1}(F \cup S)$ is irreducible then $f(x)$ is irreducible, and $f(x) \in G-F \cup S$. Certainly $f(x) \in G-F \cup S$. If $f(x)=a b$ with $a \in X Y$ and $b \in Y^{*} Z$, choose $c \in f^{-1}(a)$ and $d \in f^{-1}(b)$. Then $c \in f^{-1}(X Y)$, and $d \in f^{-1}\left(Y^{*} Z\right)$ implying $c d$ is not irreducible. 
But $f\left(x^{-1} c d\right)=1$, so $x^{-1} c d \in f^{-1}(F)$. By [14] (3.B.2.5) $x\left(x^{-1} c d\right)$ must be irreducible giving the desired contradiction.

The following theorems, 3.10-3.14, are in [14] for $G$ a finitely generated group. (With bipolar structure in 3.11-3.14.)

TheOREM 3.10. If $G$ is $\infty$-ended, then $G$ has a bipolar structure (5.A.9).

Proposition 3.11. $G_{1}=F \cup\{$ Irreducible elements of $E E\}$ and $G_{2}=F \cup\left\{\right.$ Irreducible elements of $\left.E^{*} E^{*}\right\}$ are subgroups of $G$. (3.B.4.1).

THEOREM 3.12. If $S=\varnothing$, then $G=\{F \cup S\} *_{F} G_{1}=\{F \cup S\} *_{F} G_{2}$, the free amalgamated product, (3.B.4.2) and $G$ is $\infty$-ended if and only if $F$ has index $>2$ in $G_{1}$ or equivalently $G_{2}$ (5.A.9).

THEOREM 3.13. If $S=\varnothing$ and there is no irreducible element of $E E^{*}$, then $G=G_{1} *_{F} G_{2}$ (3.B.4.3). $F$ is properly contained in $G_{1}$ and $G_{2}$ (3.B.5) and $G$ is $\infty$-ended if and only if $F$ has index $>2$ in either $G_{1}$ or $G_{2}$ (5.A.9).

THEOREM 3.14. If $S=\varnothing$ and there is an irreducible element $t$ of $E E^{*}$ then $t F t^{-1} \subset G_{1}$ and if $\phi: F \rightarrow G_{1}$ is defined by $f \rightarrow t f t^{-1}$, then $G$ is the HNN extension $G_{1_{F}} \varsigma_{\phi}$ (3.B.4.4) and $G$ is $\infty$-ended if and only if $F$ is properly embedded in $G_{1}$ (5.A.9).

REMARK 3.15. The hypothesis $S=\varnothing$ and $t$ irreducible in $E E^{*}$ also give $G=G_{2_{F}} \varsigma_{\phi}$ where $\phi: F \rightarrow G_{2}$ by $f \rightarrow t^{-1} f t$ and since $F$ is finite it can easily be shown $F$ is properly embedded in $G_{1}$ if and only if it is embedded properly in $G_{2}\left(t^{-1} G_{1} t=G_{2}\right)$.

THEOREM 3.16. If $G$ is a finitely generated $\infty$-ended group and $f: H \rightarrow G$ is an epimorphism with finite kernel then $H$ is $\infty$-ended and naturally inherits either the free product with finite amalgamation structure of Theorem 3.12 or Theorem 3.13, or the HNN structure of Theorem 3.14.

Proof. By Lemma $2.8 H$ is $\infty$-ended. By Lemma 3.8 and Theorem 3.10, $f^{-1}$ imposes a bipolar structure on $H$, determined by the one on $G$. By Lemma $3.9 h \in H$ is irreducible if and only if $f(h)$ is irreducible. Thus $f^{-1}\left(G_{1}\right)=f^{-1}(F) \cup\{$ Irreducible elements of $\left.f^{-1}(E E)\right\}$. If $S \neq \varnothing$, then $f^{-1}(S) \neq \varnothing$ and by Theorem $3.12 H=$ $\left(f^{-1}(F \cup S)\right) *_{f^{-1}(F)} f^{-1}\left(G_{1}\right)$ and $f^{-1}(F)$ has index $>2$ in $f^{-1}\left(G_{1}\right)$. By Theorem 3.13, if $S=\varnothing$ and there is no irreducible element of $E E^{*}$, then $H=f^{-1}\left(G_{1}\right) *_{f^{-1}\left(F^{\prime}\right)} f^{-1}\left(G_{2}\right), f^{-1}(F)$ is properly contained in $f^{-1}\left(G^{1}\right)$ 
and $f^{-1}\left(G_{2}\right)$, and $f^{-1}(F)$ has index $>2$ in either $G_{1}$ or $G_{2}$. If $S=\varnothing$ and there is an irreducible element $t$ of $E E^{*}$, let $u \in f^{-1}(t)$. Then by Theorem 3.14, $u f^{-1}(F) u^{-1} \subset f^{-1}\left(G_{1}\right)$ and if $\psi: f^{-1}(F) \rightarrow f^{-1}\left(G_{1}\right)$ is defined by $x \rightarrow u x u^{-1}$, then $H=f^{-1}\left(G_{1}\right) \diamond_{f^{-1}(F)} \psi$ and $f^{-1}(F)$ is properly embedded in $f^{-1}\left(G_{1}\right)$.

Corollary 3.17. If $\left\{G_{i}, f_{i}\right\}$ is an o-ended M-L-F sequence each $f_{i}$ is an epimorphism then either all $G_{i}$ have compatible free product with finite amalgamation structures as in Theorem 3.12 or Theorem 3.13, or compatible $\mathrm{HNN}$ extension structures as in Theorem 3.14.

Introduction to $\S 4$. As explained in $\S 1$ we are building the algebraic machinery necessary to extend the theorems by Hopf and Stallings from compact polyhedra $K$ to compacta $X$. If $X \equiv \lim \left\{X_{n}, f_{n}\right\}$ is a suitable compactum (this will be made precise in $\S 5$ ), $\overleftarrow{\text { we }}$ would like the Čech fundamental group $\check{\pi}_{1}(X, *) \equiv \lim \left\{\pi_{1}\left(X_{n},{ }^{*}\right), f_{n}\right\}$ to play the role of $\pi_{1}\left(K,{ }^{*}\right) . \check{\pi}_{1}\left(X,{ }^{*}\right)$ is not, in general, finitely generated, but in our situation $\stackrel{\sim}{\pi}_{1}\left(X,{ }^{*}\right)$ considered as a topological group will be compactly generated. When thus interpreted, it plays the correct role.

That is one reason for the theory of ends of M-L-F topological groups which follows.

Another is that an alternative theory of ends for compactly generated, locally compact topological groups exists, at least in part (C. Pugh and M. Shub: Axiom A actions. Inventions Math., 29 (1975), 18-31). Although we do not prove this alternative definition agrees with our "number of ends" for M-L-F topological groups a reader familiar with Pugh and Shub can check the details.

\section{Applications to topological groups.}

Definition. An M-L-F topological group is a locally compact, compactly generated, complete, metrizable topological group with countable neighborhood-basis of the identity consisting of closed and open normal subgroups.

A group can be regarded as a discrete topological group. If $\left\{G_{n}, f_{n}\right\}$ is an inverse sequence of discrete topological groups, then $\lim \left\{G_{n}, f_{n}\right\}$ in the category of topological groups is obtained by tak-

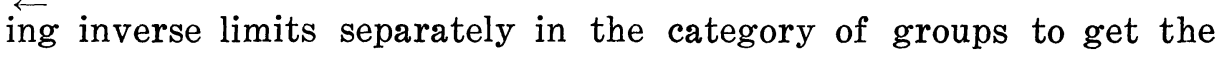
group structure, and in the category of spaces to get the topology. Throughout this section we will regard $\lim \left\{G_{n}, f_{n}\right\}$ as a topological group. 
Proposition 4.1. $G$ is an M-L-F topological group if and only if $G=\lim \left\{G_{n}, f_{n}\right\}$, where $\left\{G_{n}, f_{n}\right\}$ is an M-L-F sequence of finitely

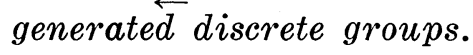

REMARK 4.1.1. For the analogous proposition for M-L groups see [1] p. 4 and [6] p. 117-8.

Proof of "only in". Let $\left\{I_{n}\right\}$ be a neighborhood-basis of the identity consisting of closed and open normal subgroups. We may assume that the $I_{n}$ are compact since $G$ is locally compact and that the $I_{n}$ are nested. Let $\pi_{n}: G \rightarrow G / I_{n}$ be the quotient homomorphism taking $g$ to its right coset $[g]$. For $U \subset G / I_{n}, \pi_{n}^{-1}(U)=\cup\left\{I_{n} x \mid x \in\right.$ $\left.\pi_{n}^{-1}(U)\right\}$. Since $I_{n} x$ is open for any $x \in G, \pi_{n}^{-1}(U)$ is open in $G$; therefore $\pi_{n}$ is continuous when $G / I_{n}$ has the discrete topology. Since $I_{n}$ is compact the obvious epimorphism $f_{n}: G / I_{n+1} \rightarrow G / I_{n}$ has kernel $\pi_{n+1}\left(I_{n}\right)$ a compact and therefore a finite group. If $K$ is a compact set which generates $G$, then $\pi_{n}(K)$ is a finite generating set in $G / I_{n}$. $\left\{G / I_{n}, f_{n}\right\}$ is M-L-F and $\lim \left\{G_{n} / I_{n}, f_{n}\right\}$ is isomorphic to $G$.

Proof of "if". First we need the following:

Lemma 4.1.2. If $\left\{G_{i}, f_{i}\right\}$ is M-L-F and $G=\lim \left\{G_{i}, f_{i}\right\}$ then the projection $\pi_{n}: G \rightarrow G_{n}$ is proper i.e., if $C$ is compact in $G_{n}$ then $\pi_{n}^{-1}(C)$ is compact in $G$.

Proof. Since $G_{n}$ is discrete, its compact subsets are precisely its finite subsets. Discreteness also implies that $G$ is closed in $\prod_{i=1}^{\infty} G_{i} . \quad \pi_{n}(C)=\left(f_{1} \circ \cdots \circ f_{n-1}(C) \times f_{2} \circ \cdots \circ f_{n-1}(C) \times \cdots \times C \times f_{n}^{-1}(C) \times\right.$ $\left.f_{n+1}^{-1} f_{n}^{-2}(C) \times \cdots\right) \cap G$. Since each $f_{i}$ has finite kernel each term in this product is finite and $\pi_{n}^{-1}(C)$ is compact, proving 4.1.2.

Now assume $G$ and $\left\{G_{n}, f_{n}\right\}$ as in Lemma 4.1.2. Since inverse limits of pro-isomorphic sequences of discrete groups are isomorphic topological groups, we may assume each $f_{n}$ is onto. If $K$ is a finite generating set of $G_{1}$, containing the identity, then by Lemma 4.1.2. $\pi_{1}^{-1}(K)$ is compact. We show $\pi_{1}^{-1}(K)$ generates $G$. If $\left(g_{1}, g_{2}, \cdots\right) \in G$ and $g_{1}=k_{1} k_{2} \cdots k_{n}$ with each $k_{i} \in K$ then since each $f_{i}$ is an epimorphism there is an $h_{i} \in G$ such that $\pi_{1}\left(h_{i}\right)=k_{i}$ for each $i . h_{1} \cdots h_{n}$ is in the subgroup of $G$ generated by $\pi_{1}^{-1}(K)$. Since $\pi_{1}\left(\left(g_{1}, g_{2}, \cdots\right)\right.$. $\left.h_{n}^{-1} \cdots h_{1}^{-1}\right)=$ identity $\in K,\left(g_{1}, g_{2}, \cdots\right)$ is in the subgroup of $G$ generated by $\pi_{1}^{-1}(K)$.

$I_{n}=\left\{\left(x_{i}\right) \in \lim \left\{G_{n}, f_{n}\right\} \mid x_{n}=\right.$ identity $\}$ forms a countable neighborhood-basis of the identity consisting of closed and open normal subgroups. By Lemma 4.1.2 each $I_{n}$ is compact and thus $G$ is locally 
compact. Each $G_{i}$ is discrete so $G$ is metrizable and as an easy exercise $G$ is complete.

DEFINITION. If $G$ is an M-L-F group the number of ends of $G$ is the number of ends of any M-L-F sequence $\left\{G_{n}, f_{n}\right\}$ where $G=\lim \left\{G_{n}, f_{n}\right\}$.

This definition is unambiguous: M-L sequences which have isomorphic (topological group) inverse limits are pro-isomorphic, see [1] and [6]; combine this with 3.1.

Many of the theorems on ends for finitely generated groups can be generalized to theorems for M-L-F groups if the word finite is replaced by compact. The following is merely an exercise in the definitions.

Proposition 4.2. An M-L-F group is 0-ended if and only if it is compact.

Definition. A closed subgroup, $H$, of a topological group $G$ has compact index in $G$ if the space of right cosets, $G / H$, with quotient topology is compact.

Proposition 4.3. An M-L-F group $G$ is 2-ended if and only if $G$ has a closed infinite cyclic subgroup of compact index.

The proof of "only if" will be done as a sequence of lemmas. Let $\left\{G_{i}, f_{i}\right\}$ be an M-L-F sequence with each $G_{i}$ 2-ended and each $f_{i}$ an epimorphism such that $G=\lim \left\{G_{i}, f_{i}\right\}$. By Proposition 3.5 there are $x_{n} \in G_{n}$ generating infinite $\overleftarrow{c y c l i c}$ subgroups of finite index in $G_{n}$ such that $f_{n}\left(x_{n+1}\right)=x_{n} \cdot x=\left(x_{1}, x_{2}, \cdots\right) \in\left\{G_{i}, f_{i}\right\}=G$.

Lemma 4.3.1. $Z_{x}=\left\{x_{1}^{n}, x_{2}^{n}, \cdots\right) \mid n$ is an integer $\}$ is a closed discrete subgroup of $\prod_{i=1}^{\infty} G_{i}$.

Proof. Say $Z_{x}$ accumulated at $y=\left(y_{1}, y_{2}, \cdots\right)$. Since $G_{i}$ is discrete $\left\{y_{1}\right\} \times G_{2} \times G_{3} \times \cdots$ is open in $\prod_{i=1}^{\infty} G_{i}$ and thus must contain an infinite number of the $x^{n}$. Each must have first coordinate $y_{2}$, but at most one can.

A similar argument shows;

Lemma 4.3.2. $G$ is closed in $\prod_{i=1}^{\infty} G_{i}$.

Lemmas 4.3.1 and 4.3.2 imply

Lemma 4.3.3. $Z_{x}$ is a closed discrete subgroup of $G$. 
Let $Z_{i}$ be the infinite cyclic subgroup of $G_{i}$ generated by $x_{i}$. Say $G_{i} / Z_{i}=\left[w_{i, 1}\right],\left[w_{i, 2}\right], \cdots,\left[w_{i, m(i)}\right] . f_{i}$ maps the elements of the right coset $\left[w_{i+1, k}\right]$ bijectively onto $\left[f_{i}\left(w_{i+1,1}\right)\right]$. Here $\left[w_{i+1, k}\right]=\left\{w_{i+1, k} x_{i+1}^{n} \mid n\right.$ is an integer $\}$, and $w_{i+1, k}$ is called a representative of $\left[w_{i+1, k}\right] . \quad \mathrm{By}$ first selecting $w_{1,1}, \cdots, w_{1, m(1)}$ and then $w_{2,1}, w_{2,2}, \cdots, w_{2, m(2)}$ etc. we may assume without loss of generality that the above selected representatives of right cosets are mapped by the appropriate bonds to other selected representatives of right cosets. I.e., $f_{n}\left(w_{n+1, k}\right) \in$ $\left\{w_{n, 1}, w_{n, 2}, \cdots, w_{n, m(n)}\right\}$ for all $k$. $W \equiv \prod_{k=1}^{\infty}\left\{w_{k, 1}, w_{k, 2}, \cdots, w_{k, m(k)}\right\}$ is compact in $\prod_{k=1}^{\infty} G_{k}$. By Lemma 4.3.2 $W \cap G$ is compact.

\section{LeMmA 4.3.4. $G / Z_{x}$ is compact.}

Proof. It suffices to show $\pi(W \cap G)=G / Z_{x}$ where $\pi: G \rightarrow G / Z_{x}$ is projection. If $[a] \in G / Z_{x}, a=\left(a_{1}, a_{2}, \cdots\right) \in G$, then let $a_{1}=w_{1, k(1)} x_{1}^{s}$. Because of how we selected representatives we have $a_{n}=w_{n, k(n)} x_{n}^{t}$ where $f_{1} \circ \cdots \circ f_{n-1}\left(w_{n, k(n)}\right)=w_{1, k(1)}$. Also, since $f_{1} \circ \cdots \circ f_{n-1}\left(a_{n}\right)=a_{1}$ we have $f_{1} \circ \cdots \circ f_{n-1}\left(x_{n}^{t}\right)=x_{1}^{s}$ and therefore $t=s$. Thus, $\pi\left(\left(w_{1, k(1)}, w_{2, k(2)}, \cdots\right)\right)=$ $\pi\left(\left(w_{1, k(1)}, w_{2, k(2)}, \cdots\right)\left(x_{1}^{s}, x_{2}^{s}, \cdots\right)\right)=\pi(a)=[a]$.

For the "if" part assume $G=\lim \left\{G_{n}, f_{n}\right\}$ where $\left\{G_{n}, f_{n}\right\}$ is M-L-F and each $f_{n}$ is an epimorphism. $\leftarrow$ Let $x=\left(x_{1}, x_{2}, \cdots\right)$ generate $Z_{x}$ a closed infinite cyclic subgroup of compact index in $G$. Let $\pi_{i}: G \rightarrow G_{i}$ be the projection morphism: $Y_{i}$ the subgroup of $G_{i}$ generated by $x_{i}$; and let $\theta_{i}: G / Z_{x} \rightarrow G_{i} / Y_{i}$ be defined by $[w] \rightarrow\left[w_{i}\right]$, where $w=\left(w_{1}, w_{2}, \cdots\right)$. To see $\theta_{i}$ is a well-defided function, let $z \in[w]$ i.e., $z=w x^{n}$. Then $z_{i}=w_{i} x_{i}^{n}$ and $z_{i} \in\left[w_{i}\right]$. Since each $f_{i}$ is onto, $\pi_{i}$ and $\theta_{i}$ are onto. Topologize $G_{i} / Y_{i}$ and $G / Z_{x}$ with the quotient topologies of the projections $\alpha_{i}: G_{i} \rightarrow G_{i} / Y_{i}$ and $\alpha_{x}: G \rightarrow G / Z_{x}$, respectively. Diagram (C) commutes on the level of functions.

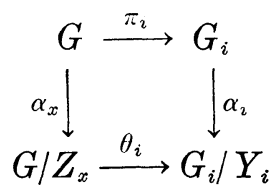

By Dugundji [4] (p. 126) $\theta_{i}$ is continuous. Since $G_{i}$ has the discrete topology and $G / Z_{x}$ is compact, $G_{i} / Y_{i}$ has the discrete topology and is compact i.e., $G_{i} / Y_{i}$ is finite. If $x_{i}$ has finite order, then since each $f_{n}$ has finite kernel each $x_{n}$ would have finite order. This implies $Z_{x}$ accumulates at the identity, contrary to the assumption $Z_{x}$ is a closed infinite cyclic subgroup of $G$. Thus each $x_{i}$ generates an infinite cyclic subgroup of finite index in $G_{i}$.

As we remarked in $\S 3$, every finitely generated group with two ends has a normal infinite cyclic subgroup of finite index. One 
might expect a normal infinite cyclic subgroup of compact index in our 2-ended M-L-F groups. But this is not the case.

ExAMPLE 4.3. The 2-ended M-L-F group, $G$, determined by Example 3.6 contains no normal infinite cyclic subgroup.

Recall that $G_{1}$ was infinite cyclic with generator $x_{1}$. The closing argument of Example 3.6 shows that $\left(b_{1}, b_{2}, \cdots\right) \in G$ cannot generate a normal infinite cyclic subgroup if $b_{1}=x_{1}^{m}$ for $m \neq 0$. But if $m=0$ then $\left(b_{1}, b_{2}, \cdots\right)$ has order two. Thus $G$ has no normal infinite cyclic subgroup.

Proposition 4.4. If $G$ is a 2-ended M-L-F group, then $G$ contains a compact open normal subgroup $F$ such that $G / F$ is $Z$ or $Z_{2} * Z_{2}$.

Proof. By Proposition $4.1 G$ is the inverse limit of an M-L-F sequence $\left\{G_{n}, f_{n}\right\}$ of 2-ended groups. Choose $F_{1}$ a finite normal subgroup of $G_{1}$ such that $G_{1} / F_{1}$ is $Z$ or $Z_{2} * Z_{2}$ ([14] p. 38). If $\pi_{1}: G \rightarrow G_{1}$ and $\pi: G_{1} \rightarrow G_{1} / F_{1}$ are projections then $\operatorname{ker}\left(\pi \circ \pi_{1}\right)=\pi_{1}^{-1}\left(F_{1}\right)$. By Lemma 4.1.2 $\pi_{1}^{-1}\left(F_{1}\right)$ is compact and open. Since $\pi_{1}^{-1}\left(F_{1}\right)=\operatorname{ker}\left(\pi \circ \pi_{1}\right), \pi_{1}^{-1}\left(F_{1}\right)$ is normal in $G$. Finally $G_{1} / F_{1}=G / \operatorname{ker}\left(\pi \circ \pi_{1}\right)=G / \pi_{1}^{-1}\left(F_{1}\right)$.

Next we classify the $\infty$-ended M-L-F groups.

REMARK 4.5. In a bipolar structure the requirement that $F$ and $S$ be finite is strictly a requirement to prove theorems about ends. Nowhere is the finiteness of $F$ and $S$ used in Stallings [14] p. 31-34. In particular it is not used in the first parts of our Theorems 3.123.14, nor is it used in Lemmas 3.8 and 3.9, and Proposition 3.11. Thus if $H$ is a group with bipolar structure (not necessarily a finiteness condition on $F$ and $S$ ) and $f: G \rightarrow H$ is an epimorphism then $f^{-1}$ induces a bipolar structure (possibly without finiteness condition) on $G$, and thus the corresponding amalgamated free product or HNN extension structure on $G$.

Theorem 4.6. The M-L-F group $G$ is $\infty$-ended if and only if $G$ has subgroups $A, B$ and $C$ such that $G$ is (in the obvious manner) isomorphic, in the category of groups, to $A{ }_{C} B$ where $C$ is compact and open in $G$, properly contained in $A$ and $B$ and of index $\geqq 3$ in either $A$ or $B$; or $G$ is isomorphic, in the category of groups, to $H \varsigma_{C} \phi$ where $C$ is compact and open in $G$ and the infinite cyclic subgroup of $H \varsigma_{C} \phi$ generated by the extra generator $x$ is closed in $G$. 
Proof of "only if". Assume $G=\lim \left\{G_{i}, f_{i}\right\}$ where each $G_{i}$ is $\infty$-ended and each $f_{i}$ is an epimorphism. If $G_{1}=A_{1} *_{C_{1}} B_{1}$ as in Theorem 3.12 or 3.13 then by Remark $4.5 G=\pi_{1}^{-1}(A) * \pi_{1}^{-1}(B) \pi_{1}^{-1}\left(C_{1}\right)$ where $\pi_{1}: G \rightarrow G_{1}$ is projection. If $G_{1}=H_{1} \varsigma_{C_{1}} \phi_{1}$ as in Theorem 3.1, with $t$ irreducible in $E E^{*}$ then by Remark $4.5 G=\pi_{1}^{-1}\left(H_{1}\right) \varsigma_{\pi_{1}^{-1}\left(C_{1}\right)} \phi$ where $q \in \pi_{1}^{-1}(t)$ and $\phi: \pi_{1}^{-1}\left(C_{1}\right) \rightarrow \pi_{1}^{-1}\left(H_{1}\right)$ by $y \rightarrow q y q^{-1}$. By Lemma 4.1.2 $\pi_{1}^{-1}\left(C_{1}\right)$ is compact in both cases and is open since $\pi_{1}$ is continuous. The infinite cyclic subgroup of $G$ generated by $q$ is closed in $G$ by Lemma 4.3.1.

\section{Proof of "if".}

Case 1. $G=A *_{C} B$ as above.

Assume $G=\lim \left\{G_{i}, f_{i}\right\}$ with each $f_{i}$ an epimorphism. Let $\pi_{n}: G \rightarrow G_{n}$ be projection. By Lemma 4.1.2 $\operatorname{ker}\left(\pi_{n}\right)$ is compact. $\bigcap_{i=1}^{\infty} \operatorname{ker}\left(\pi_{i}\right)=(e, e, \cdots)$, the identity of $G$. Since $C$ is open and $G$ is metrizable, $\operatorname{ker}\left(\pi_{n}\right) \subset C$ for some $N$. We show for this $N$ that $G_{n}$ is the free product with finite amalgamation $A_{n} *_{C_{n}} B_{n}$, where $A_{n}=$ $\pi_{n}(A), B_{n}=\pi_{n}(B)$ and $C_{n}=\pi_{n}(C)$. Since $G_{n}$ is discrete, $\pi_{n}$ is continuous and $C$ is compact, $C_{n}$ is finite.

Define $\phi_{n}: A_{n} *_{C_{n}} B_{n} \rightarrow G_{n}$ to be the homomorphism which is an inclusion on $A_{n} \cup B_{n}$. Define $\psi_{n}: A *_{C} B \rightarrow A_{n} *_{c_{n}} B_{n}$ to be the epimorphism which is projection into the $n$th coordinate on $A \cup B$.

The following diagram (D) commutes:

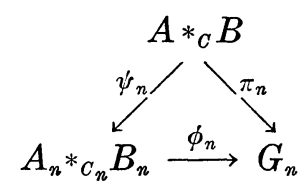

$\pi_{n}$ is an epimorphism since each $f_{i}$ is an epimorphism. Thus $\dot{\phi}_{n}$ is an epimorphism. It remains to show $\phi_{n}$ is a monomorphism. If $\phi_{n}(x)=e$, choose $y \in \psi_{n}^{-1}(x) . \quad y \in \operatorname{ker}\left(\pi_{n}\right) \subset C$, and thus $x \in C_{n}$. By the definition of $\phi_{n}, x=e$.

We have proved $G_{m}=A_{m} *_{m} B_{m}$ for all $m \geqq N$. Choose $k \geqq N$ large enough to ensure $C_{k}$ has index $\geqq 2$ in $A_{k}$ and $B_{k}$ and index $\geqq 3$ in either $A_{k}$ or $B_{k}$. Then $G_{k}=A_{k} *_{C_{k}} B_{k}$ is $\infty$-ended and by Proposition 2.4 all $G_{i}$ are $\infty$-ended.

Case 2. $G=H \varsigma_{C} \phi$ as above.

Recall $H \varsigma_{C} \phi=\left\langle H, x \mid x^{-1} c x=\phi(c) \forall c \in C\right\rangle$. As in Case 1, $\operatorname{ker}\left(\pi_{n}\right) \subset C$ for some $N$. Let $H_{n}=\pi_{n}(H), C_{n}=\pi_{n}(C)$ and $x_{n}=\pi_{n}(x)$. For $a$ and $b$ in $C$ if $\pi_{n}(a)=\pi_{n}(b)$, then $\pi_{n}\left(x^{-1} a x\right)=\pi_{n}\left(x^{-1} b x\right)$ and $\pi_{n}(\dot{\phi}(a))=\pi_{n}(\dot{\phi}(b))$. Thus $\phi_{n}: C_{n} \rightarrow H_{n}$ by $c_{n} \rightarrow \pi_{n}(\phi(c))$ where $\pi_{n}(c)=c_{n}$ 
is well-defined. In forming $H_{n} \varsigma_{c_{n}} \phi_{n}$ we identify $x_{n}^{-1} c_{n} x_{n}$ with $\phi_{n}\left(c_{n}\right)$ for all $c_{n} \in C_{n}$. If we define $\psi_{n}: H \cup\{x\} \rightarrow H_{n} \hookrightarrow_{c_{n}} \phi_{n}$ by $\psi_{n}=\pi_{n}$ then $\psi_{n}$ extends to an epimorphism of $H \varsigma_{c} \phi$ since $\psi_{n}\left(x^{-1} c x\right)=x_{n}^{-1} c_{n} x_{n}=$ $\phi_{n}\left(c_{n}\right)=\pi_{n}(\phi(c))=\psi_{n}(\phi(c))(\phi(c) \in H)$. Define $\alpha_{n}: H_{n} \hookrightarrow_{C_{n}} \phi_{n} \rightarrow G_{n}$ to be the homomorphism which extends the inclusion of $H_{n} \cup\left\{x_{n}\right\}$ into $G_{n}$.

The following diagram (E) commutes:

(E)

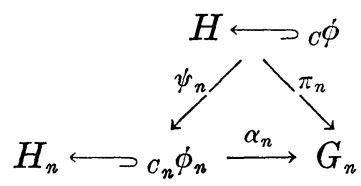

The same closing argument as that of Case 1 works here to complete the proof.

THEOREM 4.7. If $G=A *_{c} B$ as in Theorem 4.6, then in the following diagram the outer square commutes; furthermore, given any topological group $H$ and continuous homomorphisms $f: A \rightarrow H$ and $g: B \rightarrow H$ making the north-west triangle commute the resulting homomorphism of groups, $h$, (which exists and is unique by the universal property for amalgamated free products in groups) is continuous. (Unlabeled maps are inclusions.)

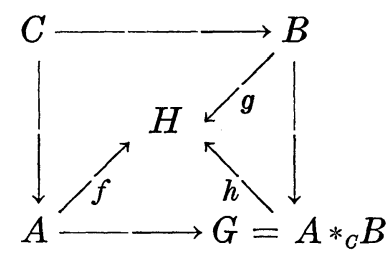

Proof. Let $W$ be a neighborhood of $x$ in $H$. Let $y \in h^{-1}(x) \subset$ $h^{-1}(W) \cdot h^{-1}(W)=h^{-1}\left(W x^{-1}\right) y$. Since $C$ is open in $G, A$ is open in $G$. Hence $f^{-1}\left(W x^{-1}\right)$ is a neighborhood of $e$ lying in $h^{-1}\left(W x^{-1}\right)$. Thus $f^{-1}\left(W x^{-1}\right) y$ is a neighborhood of $y$ lying in $h^{-1}(W)$. So $h^{-1}(W)$ is open.

REMARK 4.8. If $G=H \varsigma_{c} \phi$ as in Theorem 4.6 a similar result is true.

5. Geometric applications. As explained in $\S 1$, we will now use the theorems of $\S 3$ to derive a new shape invariant for a large class of compacta (those with M-L-F "fundamental pro-group"), and a natural geometric interpretation of this invariant, (namely the number of ends of universal covers of compact polyhedra in an associated inverse sequence). For a somewhat smaller class of compacta (those with stable "fundamental pro-group") an added na- 
tural geometric interpretation arises. We will use [5] as our reference for shape theory.

We deal throughout with a pointed connected compactum $(X, *)$, [Convention: "compactum means compact metric space. $*$ is used for all base points.] We will call a compactum, $X$, an M-L-F compactum if $X$ is connected and if for some (hence any) $* \in X$, pro- $\pi_{1}(X, *)$ is M-L-F.

The shape invariant for M-L-F compacta $X$ mentioned above can now be defined. It is $e(X) \equiv$ the number of ends of pro- $\pi_{1}(X, *)$; see $\S 3 e(X)$ is one of the numbers $0,1,2$ or $\infty$.

Theorem 5.1. If $X$ is an M-L-F compactum then $(X, *)=$ $\lim \left\{\left(X_{n}, *\right), f_{n}\right\}$ where each $X_{n}$ is a compact polyhedron and the universal cover of $X_{n}$ has $e(X)$ ends for all $n$.

Proof. By a trick of Krasinciewicz [11] (or see Theorem 4 of [7]) one can arrange $(X, *)=\lim \left\{\left(X_{n}, *\right), f_{n}\right\}$ where each $X_{n}$ is a compact polyhedron and $f_{n \sharp}$ is an epimorphism. By Hopf's theorem (see $\S 1$ ) and Proposition 2.4 the universal cover of $X_{n}$ has $e(X)$ ends for all $n$.

Note that if $(Y, *)$ is an M-L-F compactum then by a theorem of Krasinciewicz (see [7]) $(Y, *)$ is pointed shape equivalent to some $\left(X,{ }^{*}\right)$ where $X$ is compact connected and $L C^{\circ}$. We permanently assume $\left(X,{ }^{*}\right)=\lim \left\{\left(X_{n},{ }^{*}\right), f_{n}\right\}$ to have these properties and we assume each $f_{n \ddagger}$ is an epimorphism on $\pi_{1}$.

It remains to investigate when $e(X)$ can be interpreted geometrically as the number of topological ends of a locally compact space $\bar{X}$ (which reduces to the universal cover when $X$ is $L C^{\circ}$ and semilocally 1-connected). The $\bar{X}$ we have in mind is $\lim \left\{\widetilde{X}_{n}, \widetilde{f}_{n}\right\}$. More precisely $\sim$ denotes the "pointed universal cover functor" and in the following commutative diagram $(G)$ the limit $p$ of covering projections, $p_{n}$, is a fibration with unique path lifting:

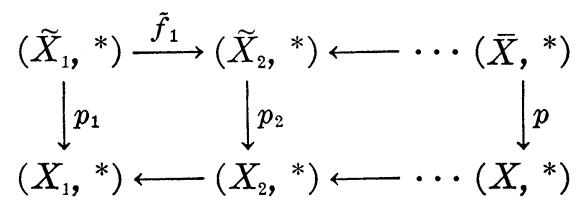

Since $X$ is M-L-F, $\bar{X}$ is locally compact. To prove this we need:

Proposition 5.2. Let $X$ and $Y$ be finite complexes. If $f: X \rightarrow Y$ induces $f_{\sharp}: \pi_{1}\left(X,{ }^{*}\right) \rightarrow \pi_{1}\left(Y,{ }^{*}\right)$ a homomorphism with finite kernel then $\tilde{f}: \tilde{X} \rightarrow \tilde{Y}$ is proper. 
Proof. Diagram (H) commutes.

(H)

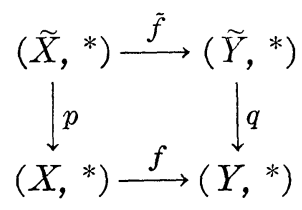

Let $[g] \in \pi_{1}\left(X,{ }^{*}\right)$, then the covering transformation of $\tilde{X}$ determined by $[g]$ is defined as follows:

If $x \in \widetilde{X}$ and $\lambda$ is a path from $x$ to ${ }^{*}$, then $p(\lambda) \cdot g \cdot p\left(\lambda^{-1}\right)$ forms a loop at $p(x) .[g](x)$ is the endpoint of the lift of this loop to $x$. By [3] (p. 12) we have:

$$
\widetilde{f}([g](x))=f_{\sharp}([g])(\widetilde{f}(x)) .
$$

Let $C$ be a compact subset of $Y$. For each cell $e_{i}$ in $X$ choose a cell $\widetilde{e}_{i}$ in $\tilde{X}$ over $e_{i}$. Then each of the cells of $\tilde{X}$ over $e_{i}$ is $h\left(\widetilde{e}_{i}\right)$ for some $h \in \pi_{1}\left(X,{ }^{*}\right)$. It suffices to show only finitely many cells of $\widetilde{X}$ over any cell, $e_{i}$, of $X$ touch $\tilde{f}^{-1}(C)$. Suppose $\left\{g_{1}, g_{2}, \cdots\right\} \subset$ $\pi_{1}\left(X,{ }^{*}\right)$ and $g_{i}\left(\widetilde{e}_{n}\right)$ intersects $\widetilde{f}^{-1}(C)$ for all $i$. Then by (1) $f_{\sharp}\left(g_{i}\right)\left(\widetilde{f}\left(\widetilde{e}_{n}\right)\right)$ intersects $C$ for each $i$. $\widetilde{f}\left(\widetilde{e}_{n}\right)$ meets only finitely many cells of $\tilde{Y}$ since the closure of $\widetilde{e}_{n}$ is compact. Thus since $f_{\sharp}$ is finiteto-one, there is a cell $\widetilde{e}$ of $\widetilde{Y}$ and infinitely many $h \in \pi_{1}\left(Y,{ }^{*}\right)$ such that $h(\widetilde{e})$ meets $C$, contradicting the local finiteness of $\widetilde{Y}$.

Let $\alpha_{i}: X \rightarrow X_{i}$ and $\widetilde{\alpha}_{i}: \bar{X} \rightarrow \widetilde{X}_{i}$ be projections. Then $p_{\imath} \circ \widetilde{\alpha}_{i}=\alpha_{i} \circ p$ for all $i, \bar{X}$ is closed in $\prod_{i=1}^{\infty} \widetilde{X}_{i}$ and since each $\widetilde{f}_{i}$ is proper, each $\widetilde{\alpha}_{i}$ is proper (see the proof of 4.1.2) and $\bar{X}$ is locally compact.

Next we discuss when $\bar{X}$ is path connected. [Certainly $\bar{X}$ can have infinitely many path components when $X$ is not $L C^{\circ}$; an example is the compact spiral:

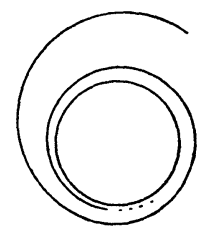

We leave it to the reader to check this.]

Proposition 5.3. $\bar{X}$ is path connected if and only if the natural $\operatorname{map} j: \pi_{1}\left(X,{ }^{*}\right) \rightarrow \lim \left\{\pi_{1}\left(X_{i},{ }^{*}\right)\right\}$ is onto.

Proof. We look at the last few terms of the homotopy exact sequence of the fibration $F \stackrel{i}{\rightarrow} \bar{X} \stackrel{p}{\rightarrow} X$ : 


$$
\begin{aligned}
& \pi_{1}\left(X,{ }^{*}\right) \stackrel{\partial}{\longrightarrow} \pi_{0}\left(F,{ }^{*}\right) \stackrel{i_{\sharp}}{\longrightarrow} \pi_{0}\left(\bar{X},{ }^{*}\right) \stackrel{p_{\sharp}}{\longrightarrow} 0 \\
& \quad \underset{\downarrow}{\lim }\left\{\pi_{1}\left(X_{i},{ }^{*}\right)\right\} .
\end{aligned}
$$

Since $F$ is totally disconnected the above isomorphism of $\lim \left\{\pi_{1}\left(X_{i},{ }^{*}\right)\right\}$ to $\pi_{0}\left(F,{ }^{*}\right)$ ls induced by the above homeomorphism of $\overleftarrow{\lim }\left\{\pi_{1}\left(X_{i},{ }^{*}\right)\right\}$ to $F$. It remains to observe the following are all $\overleftarrow{\text { equivalent: }}$

(i ) $j$ is onto

(ii) $\partial$ is onto

(iii) $\pi_{0}(\bar{X}, *)$ is trivial

(iv) $\bar{X}$ is path connected.

Proposition 5.4. Let $X$ be an $L C^{\circ}$ compactum and let pro- $\pi_{1}$ $\left(X,{ }^{*}\right)$ be stable. Then $j: \pi_{1}\left(X,{ }^{*}\right) \rightarrow \lim _{\leftarrow}\left(\right.$ pro- $\left.\pi_{1}\left(X,{ }^{*}\right)\right)$ is onto.

Proof. Let $\left\{U_{n}\right\}_{n=1}^{\infty}$ be a nested sequence of compact $Q$-manifold neighborhoods of $X$ with $\bigcap_{n=1}^{\infty} U_{n}=X$. Let $\left\{\left[W_{n}\right]\right\}$ be an element of $\lim \left\{\pi_{1}\left(U_{n},{ }^{*}\right)\right\}$. (Here there is a common base point ${ }^{*} \in X$.) Then $W_{n+1}^{\leftarrow}$ is a loop in $U_{n+1}$ which is homotopic rel. $\{0,1\}$ to $W_{n}$ in $U_{n}$. By [5] (p. 94), for any $n>0$ there is a $M$ such that for all $m>M$ $W_{m}$ is homotopic rel. $\{0,1\}$ in $U_{n}$ to a loop $\lambda_{n}$ in $X_{n}$, and hence $\lambda_{n} \cong W_{n}$ rel. $\{0,1\}$ in $U_{n}$. Let $\left\{\left[W_{n}\right]\right\} \in \lim \left\{\pi_{1}\left(U_{n},{ }^{*}\right)\right\}$ and $\lambda$ be a loop in $X$, such that $\lambda \cong W_{1}$ rel. $\{0,1\}$ in $\overleftarrow{U_{1}}$. By stability we may assume the inclusion of $U_{n+1}$ into $U_{n}$ induces an isomorphism on fundamental groups and thus $\lambda \cong W_{n}$ rel. $\{0,1\}$ in $U_{n}$ for all $n$, finishing the proposition.

Proposition 5.5. If $X$ is an $L C^{\circ}$ semi-locally 1-connected compactum then pro- $\pi_{1}\left(X,{ }^{*}\right)$ is stable.

Proof. Let $\left\{U_{n}\right\}_{n=1}^{\infty}$ be a nested sequence of compact $Q$-manifold neighborhoods of $X$ with $\bigcap_{n=1}^{\infty} U_{n}=X$. By [6] it suffices to show $\lim \left\{\pi_{1}\left(U_{n},{ }^{*}\right)\right\}$ is discrete. Assume $\left\{\left[W_{n}^{(1)}\right]\right\},\left\{\left[W_{n}^{(2)}\right]\right\}, \cdots$ are elements of $\lim _{\leftarrow}\left\{\pi_{1}\left(U_{n}, *\right)\right\}$ converging to $\left\{\left[W_{n}\right]\right\}$. By [5] (p.94) we may assume $W_{n}$ and $W_{n}^{(k)}$ are loops in $X$ for all $n$ and $k$. For any $N>0$ there is a $K(N)>0$ such that for all $k>K(N)$, and $n>N W_{n}^{(k)} \cong W_{n}$ rel. $\{0,1\}$ in $U_{N}$. Since $X$ is compact and semi-locally 1-connected there exist $\varepsilon>0$ such that any loop in $X$ of diameter $<\varepsilon$ is homotopically trivial in $X$. By [5] (p.94) there is a neighborhood $V$ of $X$ in $U_{1}$ such that any map $f:\left(L, L_{0}\right) \rightarrow(V, X)$, where $L$ is a 
1-dimensional finite polyhedron and $L_{0}$ is a subpolyhedron, is $\varepsilon / 3$ homotopic rel. $L_{0}$ in $U_{1}$ to a map $g: L \rightarrow X$. Choose $N$ such that for all $n \geqq N \quad U_{n} \subset V$. Let $k>K(N)$ and $n>N$. Then $W_{n}^{(k)} \cong W_{n}$ rel. $\{0,1\}$ in $U_{N}$ and thus in $V$. Let $H:[0,1] \times[0,1] \rightarrow V$ be a homotopy of $W_{n}^{(k)}$ to $W_{n}$ rel. $\{0,1\}$ i.e., $H \mid[0,1] \times\{0\}=W_{n}^{(k)}$, $H \mid[0,1] \times\{1\}=W_{n}$ and $H(\{0,1\} \times[0,1])={ }^{*}$. Choose $a_{0}=0<a_{1}<$ $\cdots<a_{m}=1$ and $b_{0}=0<b_{1}<\cdots<b_{m}=1$ so that the diameter $H\left(\left[a_{i}, a_{i+1}\right] \times\left[b_{j}, b_{j+1}\right]\right)$ is $<\varepsilon / 3$ for all $i$ and $j$. Let $L=\left(\left\{a_{0}, a_{1}, \cdots, a_{m}\right\} \times\right.$ $[0,1]) \cup\left([0,1] \times\left\{b_{0}, b_{1}, \cdots, b_{m}\right\}\right)$ and $L_{0}=(\{0,1\} \times[0,1]) \cup[0,1] \times\{0,1\}$. Choose $g: L \rightarrow X$ homotopic rel. $L_{0}$ to $H \mid L . g\left(\left(\left\{a_{i}, a_{i+1}\right\} \times\left[b_{j}, b_{j+1}\right]\right) \cup\right.$ $\left.\left(\left[a_{\imath}, a_{i+1}\right] \times\left\{b_{j}, b_{j+1}\right\}\right)\right)$ is a loop of diameter $<\varepsilon$ in $X$ and thus homotopically trivial in $X$. Hence $W_{n}^{(k)}$ is homotopic to $W_{n}$ rel. $\{0,1\}$ in $X$ for all $n>N$ and $k>K(N)$ i.e., $\left\{\left[W_{n}^{(k)}\right]\right\}=\left\{\left[W_{n}\right]\right\}$ for all $k>K(N)$ and $\lim \left\{\pi_{1}\left(U_{n},{ }^{*}\right)\right\}$ is discrete.

REMARK 5.5.1. If a loop $W$ of a semi-locally-1-connected compactum $X$, represents the trivial element of pro- $\pi_{1}(X)$ then $W$ is trivial in $X$ if $X$ is $L C^{\circ}$. Here is the main theorem of $\S 5$.

THEOREM 5.6. If $\bar{X}$ can be written as the union of compact sets, $A_{n}$, with $A_{n}$ a subset of the interior of $A_{n-1}$ and any two points in $A_{n}$ can be joined by a path in $A_{n+1}$ then $\bar{X}$ has the same number of ends as $\lim _{\leftarrow}\left\{\pi_{1}\left(X_{n},{ }^{*}\right)\right\}$.

The proof will be done as a sequence of lemmas. We assume * $\in A_{1}$.

REMARK 5.6.1. For $\bar{X}$ compact this is trivial.

Lemma 5.6.2. $\bar{X}$ contains a compact set $C$ such that $p$ maps the interior of $C, \operatorname{int}(C)$, onto $X$.

Proof. By the Hahn-Mazurkiewicz theorem $X$ is a Peano curve i.e., the continuous image of $[0,1]$. Lifting this path to $\bar{X}$ gives a path whose image under $p$ is $X$. Choose an $A_{n}$ such that this path lies in $\operatorname{int}\left(A_{n}\right)$, then $A_{n}$ is the desired $C$.

Let $F_{i}=p_{i}^{-1}(*)$ and $F=p^{-1}(*)$. The usual bijections $\pi_{1}\left(X_{i}, *\right) \stackrel{\sim}{\rightarrow} F_{2}$ induce a homeomorphism $H: \lim \left\{\pi_{1}\left(X_{i},{ }^{*}\right)\right\} \stackrel{\widetilde{\longrightarrow}}{\rightarrow} F$, where $\lim \left\{\pi_{1}\left(X_{i},{ }^{*}\right)\right\}$

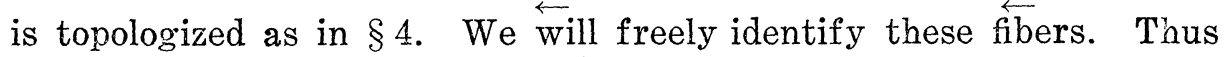
the left action of $\pi_{1}\left(X_{n},{ }^{*}\right)$ on $\widetilde{X}_{n}$ determined by the corresponding covering transformations determines a left action of $F_{n}$ on $\widetilde{X}_{n}$, hence $F$ acts on the left of $\bar{X}$ as fiber preserving homeomorphisms such that for $a \in F$ and $x \in \bar{X} \widetilde{\alpha}_{i}(a(x))=\widetilde{\alpha}_{i}(a)(x)$ for all $i$. 
For $A$ a subset of a simplicial complex $Y$ define $S t(A)$ to be the closure of the union of all cells of $Y$ that touch $A$. Inductively define $S t^{N}(A)$ to be $S t\left(S t^{N-1}(A)\right)$ for $N \geqq 2$. A set $B$ in a topological space $T$ is said to be bounded if $B$ lies in a compact subset of $T$, otherwise $B$ is unbounded.

Lemma 5.6.3. If $C$ is a compact subset of $\bar{X}$, then each unbounded path component of $\bar{X}-C$ contains an unbounded subset of $F$.

Proof. By Lemma 5.6.2 there is an $M$ such that $p\left(A_{M}\right)=X$, and $C \subset A_{M}$. Choose $N$ such that $\widetilde{\alpha}_{1}\left(A_{M+1}\right) \subset S t^{N}\left(^{*}\right)$. If $Q$ is an unbounded path component of $\bar{X}-A_{k}$, let $\left\{x_{i}\right\}_{i=1}^{\infty}$ be an unbounded collection of points in $Q-\widetilde{\alpha}_{1}^{-1}\left(S t^{4 N}\left({ }^{*}\right)\right)$. Let $y_{i} \in A_{M}$ such that $p\left(y_{i}\right)=$ $p\left(x_{i}\right)$ and let $\lambda_{i}$ be a path from $y_{i}$ to ${ }^{*}$ in $A_{M+1}$. The endpoint of the lift of $p \circ \lambda_{i}$ to $x_{i}$ is in $F$, call it $z_{i}$, thus we have: $z_{i} \cdot \lambda_{i}$ has endpoints $z_{i}$ and $x_{i}$; and $\widetilde{\alpha}_{1}\left(\mathrm{x}_{i}\right) \in \widetilde{\alpha}_{1}\left(z_{i} A_{M+1}\right) \subset S t^{N}\left(\widetilde{\alpha}_{1}\left(z_{i}\right)\right)=\alpha_{1}\left(z_{i}\right) \cdot S t^{N}\left({ }^{*}\right)$. By definition $\widetilde{\alpha}_{1}\left(x_{i}\right) \notin S t^{4 N}\left({ }^{*}\right)$, so $S t^{N}\left(\alpha_{1}\left(z_{i}\right)\right)$ contains a point of $\widetilde{X}_{1}-$ $S t^{4 N}\left(^{*}\right)$ and therefore $\widetilde{\alpha}\left(z_{i}\right) \cdot S t^{N}\left({ }^{*}\right)$ misses $S t^{N}\left(^{*}\right)$. Since their images under $\widetilde{\alpha}_{1}$ are disjoint, $z_{i} \cdot A_{M+1}$ and $A_{M+1}$ are disjoint. $z_{i} \cdot \lambda_{i} \subset z_{i} \cdot A_{M+1} \subset$ $\bar{X}-A_{M}$, therefore $z_{i} \in Q$ for all $i .\left\{z_{i}\right\}$ is unbounded, for if not, say $S_{1}\left(\left\{z_{i}\right\}\right) \subset D$, a compact set. Then $S_{1}\left(\left\{x_{i}\right\}\right) \subset S t^{N}(D)$ a compact set but $\tilde{\alpha}_{1}$ is proper and $\left\{x_{i}\right\}$ is unbounded giving the desired contradiction.

Lemma 5.6.4. If $p\left(\operatorname{int}\left(A_{M}\right)\right)=X$, then there is a bounded neighborhood of $A_{M}$ containing all but finitely many of the path components of $\bar{X}-A_{M}$.

Proof. Choose $N$ such that $\widetilde{\alpha}_{1}\left(A_{M+1}\right) \supset S t^{N}\left({ }^{*}\right)$. We show $\widetilde{\alpha}_{1}^{-1}\left(S t^{4 N}\left({ }^{*}\right)\right) \equiv W$ is the desired neighborhood of $A_{M}$. $W$ is bounded since $\widetilde{\alpha}_{1}$ is proper. Assume an infinite number of path components of $\bar{X}-A_{M}$, say $C_{1}, C_{2}, \cdots$, are not contained in $W$. Since $\bar{X}$ is path connected each of these path components meet $b d(W)$, the boundary of $W$. Choose $x_{i} \in C_{i} \cap b d(W)$. Say the $x_{i}$ accumulate at $x \in b d(W)$. Let $y \in \operatorname{int}\left(A_{M}\right)$ such that $p(y)=p(x)$. If $\lambda$ is a path from $y$ to * in $A_{M+1}$, then lifting $p \circ \lambda$ to $x$ is a path from $x$ to $z \in F$ and $z$. $A_{M+1}$ is a neighborhood of $x$ containing $z \cdot \lambda$, a path from $x$ to $z$. $\widetilde{\alpha}_{1}(x) \epsilon$ $b d\left(S t^{4 N}\left({ }^{*}\right)\right)$ and $\widetilde{\alpha}_{1}\left(z \cdot A_{M+1}\right) \subset S t^{N}\left(\widetilde{\alpha}_{1}(z)\right)=\widetilde{\alpha}_{1}(z) \cdot S t^{N}\left({ }^{*}\right)$. Thus $b d\left(S t^{4 N}\left({ }^{*}\right)\right) \cup$ $S t^{N}\left(\alpha_{1}(z)\right)$ is a nonempty subcomplex of $\widetilde{X}_{1}$, and must contain a vertex $u$. If $S t^{N}(\alpha(z)) \cap S t^{N}\left(^{*}\right) \neq \varnothing$ there would be an edge path from * to $u$ of length $\leqq 3 N$ contradicting the fact $u \in b d\left(S t^{4 N}\left({ }^{*}\right)\right)$. Hence $\widetilde{\alpha}_{1}\left(z \cdot A_{M+1}\right) \cap \widetilde{\alpha}_{1}\left(A_{M+1}\right)=\varnothing$, and $A_{M} \cap z \cdot A_{M+1}=\varnothing$. Since $z \cdot A_{M+1} \subset \bar{X}-M$ and $z \cdot A_{M}$ is a neighborhoood of $x$, the $x_{i}$ are not all from different path components of $\bar{X}-A_{M}$ giving the desired contradiction. 
Corollary 5.6.5. If $\widetilde{X}_{1}$ is $N$-ended then $\bar{X}$ has at least $N$ ends. In particular if $\widetilde{X}_{1}$ is c-ended then $\bar{X}$ is $\infty$-ended.

Proof. If $C$ is a compact subset of $\widetilde{X}_{1}$ and $\widetilde{X}_{1}-C$ has $M$ unbounded path components, then an argument analogous to the proof of Lemma 5.6.3 shows each unbounded path component, $C_{i}$, of $\widetilde{X}_{1}-C$ contains an unbounded subset, $S_{i}$, of $F_{1}$. By Lemma 5.6.4 $\bar{X}-\widetilde{\alpha}_{1}^{-1}(C)$ has only a finite number of unbounded path components and $\widetilde{\alpha}_{1}^{-1}\left(S_{2}\right)$ is an unbounded subset of $F$ since each $f_{i \ddagger}$ is an epimorphism. One of the unbounded path components of $\bar{X}-\widetilde{\alpha}_{1}^{-1}(C)$ must contain an unbounded subset of $\widetilde{\alpha}_{1}^{-1}\left(S_{i}\right)$; but $\widetilde{\alpha}_{I}^{-1}\left(S_{i}\right)$ does not meet a path component of $\bar{X}-\alpha_{1}^{-1}(C)$ which contains a point of $\widetilde{\alpha}_{1}^{-1}\left(S_{j}\right)$ for $i \neq j$. Thus $\bar{X}-\widetilde{\alpha}_{1}^{-1}(C)$ must have at least $M$ unbounded path components.

\section{Lemma 5.6.6. If $\lim \left\{\pi_{1}\left(X_{i},{ }^{*}\right)\right\}$ is 1-ended, then $\bar{X}$ is 1-ended.}

Proof. By Corollary 5.6.5 we need to see $\bar{X}$ has at most one end. Assume $\bar{X}-A^{r}$ has unbounded path components $C_{1}$ and $C_{2}$. Let $K_{1}$ be a finite set of generators of $\pi_{1}\left(X_{1},{ }^{*}\right)$ and assume $1 \in K_{1}$, then $\alpha_{1}^{-1}(K)$ is a compact generating set for $\lim \left\{\pi_{1}\left(X_{n},{ }^{*}\right)\right\}$ (see the proof of 4.1). Choose $W$ such that $K \cup K^{-1} \subset \overleftarrow{A_{W}}$. Let $M$ and $N$ be such that $\tilde{\alpha}_{1}^{-1}\left(A_{V^{+}+1}\right) \subset S t^{N}\left({ }^{*}\right)$ and $\widetilde{\alpha}_{1}\left(A_{W_{+1}}\right) \subset S t^{M}\left({ }^{*}\right)$, and hence $K_{1} \subset$ $S t^{\prime \prime}\left({ }^{*}\right)$.

Claim. It suffices to show there are elements $v_{1} \in C_{1} \cap F$ and $v_{2} \in C_{2} \cap F$ such that $v_{2}=v_{1} k_{1} k_{2} \cdots k_{n}$ where $k_{i} \in K \cup K^{-1}$ and $v_{1} k_{1} \cdots k_{m} A_{W+1} \subset \bar{X}-A_{v}$ for all $m \leqq n$.

Proof. $\quad v_{1} k_{1} \cdots k_{m} \in v_{1} k_{1} \cdots k_{m} A_{W} \cap v_{1} k_{1} \cdots k_{m+1} A_{W}$; so $v_{1} k_{1} \cdots k_{m}$ can be joined by a path to $v_{1} k_{1} \cdots k_{m+1}$ in $v_{1} k_{1} \cdots k_{m+1} A_{W^{+1}} \subset \bar{X}-A_{y}$. Thus $v_{1}$ and $v_{2}$ are in the same path component of $\bar{X}-A_{V}$ giving the desired contradiction.

Let $\widetilde{\alpha}_{1}\left(A_{V}\right) \subset S t^{N}\left(^{*}\right)$ and $\widetilde{\alpha}_{1}\left(A_{W+1}\right) \subset S t^{M}\left(^{*}\right)$. Choose $R$ such that any element of $F \cap S t^{M+N}\left(^{*}\right)$ is an $R$-fold product with factors in $K_{1} \cup K_{2}$. In $\S 2$ we define $L\left(K_{1}\right)$ which by definition has 1 -end. The verticies of $L\left(K_{1}\right)$ are identified with $\pi_{1}\left(K_{1},{ }^{*}\right)$ and thus with the elements of $F_{1}$. If $u_{1}$ and $u_{2}$ are verticies of the unbounded path component, $C$, of $L\left(K_{1}\right)-S t^{R}(1)$, then an edge path in $C$ from $u_{1}$ to $u_{2}$ gives $u_{2}=u_{1} k_{1} \cdots k_{n}$ with $k_{i} \in K_{1} \cup K_{1}^{-1}$, and for all $m \leqq n$ $u_{1} k_{1} \cdots k_{m} \in \tilde{X}_{1}-S t^{i+N}\left({ }^{*}\right)$. Since $\quad \tilde{\alpha}_{1}\left(A_{W+1}\right) \subset S t^{M I}\left(^{*}\right), \quad u_{1} k_{1} \cdots k_{m}$. $\widetilde{\alpha}_{1}\left(A_{W+1}\right) \subset \widetilde{X}_{1}-S t^{N}\left(^{*}\right)$ for all $m \leqq n$. Since $\widetilde{\alpha}_{1}$ is proper, Lemma 5.6.3 implies $C_{1}$ and $C_{2}$ contain points of $F, v_{1}$ and $v_{2}$ respectively such that $\widetilde{\alpha}_{1}\left(v_{1}\right)$ and $\widetilde{\alpha}_{1}\left(v_{2}\right)$ are verticies of $C$. Assume $\widetilde{\alpha}_{1}\left(v_{1}\right)=u_{1}$ 
and $\widetilde{\alpha}_{1}\left(v_{2}\right)=u_{2}$ as above. We can choose $h_{j} \in \widetilde{\alpha}_{1}^{-1}\left(k_{j}\right) \subset K \cup K^{-1}$ since $f_{i \sharp}$ is an epimorphism for all $i$. Since $\widetilde{\alpha}_{1}\left(A_{V}\right) \subset S t^{N}\left({ }^{*}\right), v_{1} h_{1} \cdots h_{m}$. $A_{W+1} \subset \bar{X}-A_{V}$ for all $m \leqq n$. Let $h_{n+1}=\left(v_{1} h_{1} \cdots h_{n}\right)^{-1} v_{2}$, then $\alpha_{1}\left(h_{n+1}\right)={ }^{*} \equiv 1 \in K_{1}$, and $\alpha_{1}\left(v_{1} h_{1} \cdots h_{n+1} \cdot A_{W+1}\right)=\alpha_{1}\left(v_{1} h_{1} \cdots h_{n} \cdot A_{W+1}\right) \subset$ $\widetilde{X}_{1}-\operatorname{St}^{N}\left({ }^{*}\right)$. Thus $v_{2} \cdot A_{W+1} \subset \bar{X}-A_{V}$ and by the above claim the proof is finished.

Lemma 5.6.7. If $\lim \left\{\pi_{1}\left(X_{n},{ }^{*}\right)\right\}$ is 2-ended then $\bar{X}$ is 2-ended.

Proof. By Corollary 5.6.5 we need to prove $\bar{X}$ has at most 2-ends. Let $G=\lim \left\{\pi_{1}\left(X_{i},{ }^{*}\right)\right\}$, then $G$ has a closed infinite cyclic subgroup, $Z_{x}$, with generator $x$, and a compact subset $K$ such that if $\beta$ is the quotient map of $G$ to $G / Z_{x}$ then $\beta(K)=G / Z_{x}$ (see 4.3.4). Hence $G=\cup\left\{x^{n} \cdot K \mid n\right.$ is an integer $\}, \tilde{\alpha}_{1}(K)$ is a compact subset of the discrete fiber $F_{1}$, i.e., $\widetilde{\alpha}_{1}(K)$ is finite. Thus (1) If $V$ is compact in $\bar{X}$ then $\widetilde{\alpha}_{1}\left(\bigcup_{k \in K} k \cdot V\right)$ is finite.

Let $A_{W}$ be such that $p\left(A_{W}\right)=X$ and $x \cdot K \cup K \subset A_{W}$. It suffices to show for $Q \geqq W, \bar{X}-A_{Q}$ has at most two unbounded path components. By Lemma 5.6.3 it suffices to show there exists $M>0$ and $N<0$ such that for all $m>M$ all points of $x^{m} \cdot K$ are in the same path component of $\bar{X}-A_{Q}$ and for $n<N$ all points of $x^{n}$. $K$ are in the same path component of $\bar{X}-A_{Q} . \tilde{\alpha}_{1}(x), \widetilde{\alpha}_{1}\left(x^{2}\right), \cdots$ is a closed discrete subset of $\tilde{X}_{1}$. Choose $K$ and $L$ such that $\widetilde{\alpha}_{1}\left(A_{Q}\right) \subset$ $S t^{L}\left(^{*}\right)$ and $\tilde{\alpha}_{1}\left(A_{W+1}\right) \subset S t^{K}\left(^{*}\right)$. Let $M$ be such that for all $m>M$ $\widetilde{\alpha}_{1}\left(x^{m}\right)$ misses $S t^{L+M}(*)$. Then $\widetilde{\alpha}_{1}\left(x^{m}\right) \cdot \widetilde{\alpha}_{1}\left(A_{W+1}\right)$ misses $\widetilde{\alpha}_{1}\left(A_{Q}\right)$ for all $m>M$. Thus $x^{m} \cdot A_{W+1}$ misses $A_{Q}$ for all $m>M$. $\left(x^{m} \cdot K\right) \cup\left(x^{m+1} \cdot K\right) \subset$ $x^{m} A^{W}$ so all points of $\left(x^{m} \cdot K\right) \cup\left(x^{m+1} \cdot K\right)$ can be joined by paths in $x^{m} A_{W+1} \subset \bar{X}-A_{Q}$ for all $m>M$. A similar argument holds for negative powers of $x$.

Combining Remark 5.6.1, Corollary 5.6.5 and Lemmas 5.6.6 and 5.6.7 proves Theorem 5.6.

THEOREM 5.7. Let $Y$ be a connected compactum with pro- $\pi_{1}\left(Y,{ }^{*}\right)$ stable. Then any $L C^{\circ}$ compactum $X \equiv \lim \left\{X_{n}\right\}$ in the shape class of $Y$ has the property that $e(X)$ is the number of topological ends of the path connected, locally compact space $\bar{X} \equiv \lim \left\{\widetilde{X}_{n}\right\}$. Moreover when $X$ is semi-locally 1-connected, $X$ is the universal cover of $X$.

REMARK 5.8. By [11] there exist $L C^{\circ}$ compacta in the shape class of $Y$.

Proof. We prove any compact subset of $\bar{X}$ is contained in a path connected compact subset of $\bar{X}$. By Propositions 5.3 and 5.4 and Theorem 5.6 this will prove the first part of our theorem. 
Let $\beta$ be a path in $X$ whose image covers $X$ (Hahn-Mazurkiewicz theorem), and let $C$ be a compact subset of $\bar{X}$. If $\beta^{\prime}$ is a lift of $\beta$ to $\bar{X}$. Choose $N$ such that for any $x$ in the image of $\widetilde{\alpha}_{1} \circ \beta^{\prime}$, the image of $\tilde{\alpha} \circ \beta^{\prime}$ is a subset of $S t^{N}(x)$. Let $D=C \cup\left(\bigcup_{x \in C} \beta_{x}\right)$ where $\beta_{x}$ is the image of a lift of $\beta$ containing $x$. If $\widetilde{\alpha}_{1}(C) \subset S t^{M}\left({ }^{*}\right)$ then $\alpha_{1}(D) \subset \mathrm{St}^{M+N}\left(^{*}\right)$ and $D$ is bounded. Since pro- $\pi_{1}\left(X,{ }^{*}\right)$ is stable $F$ is discrete and $F \cap D$ is finite. By Propositions 5.3 and $5.4 \bar{X}$ is path connected. Let $E$ be $D$ union a path containing each point of $F \cap D$. Then $E$ is connected and contains $C$.

For the second half of 5.7 , let $q:\left(\widetilde{X},{ }^{*}\right) \rightarrow\left(X,{ }^{*}\right)$ be the universal covering projection. There are unique maps $\beta_{n}:\left(\widetilde{X},{ }^{*}\right) \rightarrow\left(\widetilde{X}_{n},{ }^{*}\right)$ such that $p_{n} \circ \beta_{n}=\alpha_{n} \circ q \cdot \alpha_{n-1} \circ q=f_{n} \circ \alpha_{n} \circ q=f_{n} \circ p_{n} \circ \beta_{n}=p_{n-1} \circ \tilde{f}_{n-1} \circ \beta_{n}$. By the uniqueness of pointed lifts, $\beta_{n-1}=\tilde{f}_{n-1} \circ \beta_{n}$ and there is a map $\beta: \tilde{X} \rightarrow \bar{X}$ by $x \rightarrow\left(\beta_{1}(x), \beta_{2}(x), \cdots\right)$ we prove $\beta$ is a fiber preserving homeomorphism. Since $\alpha_{n} \circ q=p_{n} \circ \beta_{n}=p_{n} \circ \tilde{\alpha}_{n} \circ \beta=\alpha_{n} \circ p \circ \beta$ for all $n, p \circ \beta=q$ and $\beta$ is fiber preserving. Thus to see that $\beta$ is onto and one-to-one it suffices to show $\beta$ is onto and one-to-one on fibers. Let $x \in F \subset \bar{X}$. Since $j: \pi_{1}\left(X,{ }^{*}\right) \rightarrow \lim \left\{\pi_{1}\left(X_{n},{ }^{*}\right)\right\} \equiv F$ is onto (see Propositions 5.4 and 5.5) there is a loop $\lambda$ in $X$ such that the lift of $\lambda$ to ${ }^{*} \in \bar{X}$, call it $\lambda_{1}$, has endpoint $x$. I.e., $\lambda_{1}(0)={ }^{*}$ and $\lambda_{1}(1)=x$. Call the lift of $\lambda$ to $* \in \tilde{X}, \lambda_{2}$. Since $p \circ \beta=q$, unique path lifting in $\bar{X}$ implies $\beta \circ \lambda_{2}=\lambda_{1}$ and thus $\beta\left(\lambda_{2}(1)\right)=x$ so $\beta$ is onto.

If $\beta(x)=\beta(y)$ let $\gamma$ be a path from $x$ to $y . \beta_{n}(x)=\beta_{n}(y)$ for all $n$, thus $p_{n} \circ \beta_{n} \circ \gamma$ is a trivial loop in $X_{n}$ for all $n$. By Remark 5.5.1 $p \circ \beta \circ \gamma$ is a trivial loop in $X$ and hence $q \gamma$ is a trivial loop in $X$ implying $x=y$. I.e., $\beta$ is one-to-one.

TheOREM 5.9. Let $X$ and $Y$ be M-L-F compacta such that $\bar{X}$ is path connected and $X$ is $L C^{\circ}$. Then for any map $f:\left(X,{ }^{*}\right) \rightarrow$ $\left(Y,{ }^{*}\right)$ there is a unique map $\bar{f}:\left(\bar{X},{ }^{*}\right) \rightarrow\left(\bar{Y},{ }^{*}\right)$ such that $p \circ f=\bar{f} \circ q$. (Here $p$ and $q$ are projections.)

Proof. Let $j: \pi_{1}\left(X,{ }^{*}\right) \rightarrow \lim$ pro- $\pi_{1}\left(X,{ }^{*}\right)$ and $j^{\prime}: \pi_{1}\left(Y,{ }^{*}\right) \rightarrow \lim$ pro- $\pi_{1}\left(Y,{ }^{*}\right)$ be the natural maps. Let $\widetilde{\alpha}_{n}:\left(\bar{X},{ }^{*}\right) \rightarrow\left(\widetilde{X}_{n},{ }^{*}\right)$ be projection. If $\lambda$ is a loop at $*$ in $X$ then $\tilde{\alpha}_{n} \circ \lambda$ is a trivial loop in $\left(\widetilde{X}_{n},{ }^{*}\right)$ and thus $p_{n} \circ \tilde{\alpha}_{n} \circ \lambda=\alpha_{n} \circ p \circ \lambda$ is a trivial loop in $\left(X_{n},{ }^{*}\right.$. Hence $p_{\sharp}\left(\pi_{1}\left(\bar{X},{ }^{*}\right)\right) \subset \operatorname{ker}(j)$. A similar argument shows $\operatorname{ker}(j) \subset p_{\sharp}\left(\pi_{1}\left(\bar{X},{ }^{*}\right)\right)$ and since $p_{\#}$ is a monomorphism (see [13] p. 72), we have:

REMARK 5.9.1. $\pi_{1}\left(\bar{X},{ }^{*}\right)$ is isomorphic to $\operatorname{ker}(j)$.

Claim 1. $f_{\sharp}(\operatorname{ker}(j)) \subset \operatorname{ker}\left(j^{\prime}\right)$. 
Proof. Assume $X$ and $Y$ are embedded in the Hilbert Cube, $Q$. Since $Q$ is an absolute retract $f$ can be extended to a map $f^{\prime}: Q \rightarrow Q$. If $\lambda$ is a loop in $X$ at ${ }^{*}$, representing an element of $\operatorname{ker}(j)$ then for any neighborhood $V$ of $X$, (in $Q$ ) $\lambda$ is trivial $\operatorname{rel}\{0,1\}$ in $V$. Thus by the uniform continuity of $f^{\prime}, f \circ \lambda$ is trivial rel $\{0,1\}$ in any neighborhood of $Y$.

Let $x \in \bar{X}$ and $\lambda$ a path from $x$ to ${ }^{*}$. Define $\bar{f}(x)$ to be the end point of the lift of $f \circ p \circ \lambda$ to ${ }^{*} \in Y$. This is a well-defined function (see [13] p. 76), and it suffices to show $\bar{f}$ is continuous.

Claim 2. $\bar{f} \mid p^{-1}(x)$ is continuous for any $x \in X$.

Proof. Let $\varepsilon>0$ be given. There is a $\delta>0$ such that if $d\left(x_{1}, x_{2}\right)<\delta\left(x_{1}\right.$ and $x_{2}$ in $\left.p^{-1}(x)\right)$ and $\lambda$ is a path from $x_{1}$ to $x_{2}$ then $p \circ \lambda$ is homotopically trivial $\operatorname{rel}\{0,1\}$ in an $\varepsilon$-neighborhood of $X \subset Q$. (Assume $X$ and $Y$ are embedded in $Q$ and $f^{\prime}$ is as in Claim 1.) By the uniform continuity of $f^{\prime},\left.\bar{f}\right|_{p-1(x)}$ is continuous.

Claim 3. Let $\bar{x} \in \bar{X}$ and $\varepsilon>0$ be given. There is a $\delta>0$ such that if $\lambda$ is a path at $x \equiv p(\bar{x})$ and diam. $(\lambda)$ (The diameter of the image of $\lambda$ ) is less than $\delta$ then the lift of $\delta$ to $\bar{x}$ has diameter less than $\varepsilon$.

Proof. Define a metric, $d$, on $X$ by: $d(a, b) \equiv \sum_{i=1}^{\infty}(1 / 2)^{i}\left(d_{i}\left(a_{i}, b_{i}\right) /\right.$ $\left.\left(1+d_{i}\left(a_{i}, b_{i}\right)\right)\right)$, where $d_{i}$ is a metric on $X_{i}$ and $a_{i}=\alpha_{i}(a)$. Similarly define a bounded metric on $\bar{X}$ (also denoted by $d$ ). For an evenly covered compact neighborhood $U_{n}$ of $x_{n}$ there is a compact neighborhood $\widetilde{U}_{n}$ of $\bar{x}_{n}$ such that $p_{n} \mid \widetilde{U}_{n}$ is a uniformly continuous homeomorphism, as is its inverse. Thus for any n, paths of "small" diameter at $x_{n}$ lift (in $\widetilde{X}_{n}$ ) to paths of "small" diameter at $\bar{x}_{n}$. By the uniform continuity of $\alpha_{i}: X \rightarrow X_{i}$, for any $N>0$ and $\delta_{i}>0$ there is a $\delta_{2}\left(\delta_{1}, N\right)>0$ such that if a path $\lambda$ at $x$ has diameter less than $\delta_{2}\left(\delta_{1}, N\right)$ then diam. $\left(\alpha_{n} \circ \lambda\right)<\delta_{1}$ for all $n \leqq N$. Choose $N$ such that $(1 / 2)^{N}<\varepsilon / 2$ and $\delta_{1}$ such that if $\beta$ is a path at $x_{n}$ of diameter less than $\delta_{1}$ then the lift of $\beta$ to $\bar{x}_{n}$ is of diameter less than $\varepsilon / 2$. By our choice of metric if $\lambda$ is a path at $x$ of diameter less than $\delta_{2}\left(\delta_{1}, N\right)$ then the lift of $\lambda$ to $\bar{x}$ has diameter less than $\varepsilon$.

Now we show $\bar{f}$ is continuous at $x \in \bar{X}$. Let $\varepsilon>0$ be given. By the uniform continuity of $f$ and Claim 3 applied to $\bar{Y}$ there is a $\delta_{1}>0$ such that if $\lambda$ is a path at $p(x)$ of diameter less than $\delta_{1}$ then the lift of $f \circ \lambda$ to $\bar{f}(x)$ has diameter less than $\varepsilon / 2$. By Claim 2 there is a $\delta_{2}>0$ such that if $d(a, x)<\delta_{2}$ where $a \in p^{-1}(p(x))$ then $d(\bar{f}(a), \bar{f}(x))<\varepsilon / 2$. By Claim 3 there exists $\delta_{3}<\delta_{1}$ such that $\delta_{3}>0$ 
and if $\lambda$ is a path at $p(x)$ of diameter less than $\delta_{3}$ then the lift of $\lambda$ to $x$ has diameter less than $\delta_{2} / 2$. Since $X$ is $L C^{\circ}$ there is a $\delta_{4}>0$ such that if $b \in X$ and $d(b, p(x))<\delta_{4}$ then there is a path of diameter less than $\delta_{3}$ from $b$ to $p(x)$. By the continuity of $p$ there is a $\delta_{5}>\delta_{2} / 2$ such that $\delta_{5}>0$ and if $d(a, x)<\delta_{5}$ then $d(p(a), p(x))<\delta_{4}$. We show if $d(a, x)<\delta_{5}$ then $d(\bar{f}(a), \bar{f}(x))<\varepsilon . d(a, x)<\delta_{5}$ implies there is a path $\lambda$ from $p(a)$ to $p(x)$ of diameter less than $\delta_{3}$. Let $z \in$ $p^{-1}(p(x))$ be the end point of $\lambda$ lifted to $a, d(z, a)<\delta_{2} / 2$ and $d(a, x)<$ $\delta_{5}<\delta_{2} / 2$ so $d(z, x)>\delta_{2}$. Hence $d(\bar{f}(z), \bar{f}(x))>\varepsilon / 2$. Since diam. $(\lambda)<\delta_{3}<\delta_{1}$, the lift of $f \circ \lambda$ to $\bar{f}(a)$ has diameter less than $\varepsilon / 2$ and has end point $\bar{f}(z)$. Hence $d(\bar{f}(x), \bar{f}(a))<\varepsilon$ and $\delta_{5}$ is the desired bound.

CoRollary 5.9.2. If $f:\left(X,{ }^{*}\right) \rightarrow\left(Y,{ }^{*}\right)$ is a homeomorphism of $L C^{\circ}$ M-L-F compacta and $\bar{X}$ and $\bar{Y}$ are path connected then the induced $\operatorname{map} \bar{f}:\left(\bar{X},{ }^{*}\right) \rightarrow\left(\bar{Y},{ }^{*}\right)$ is a homeomorphism.

TheOREM 5.10. Let $f:\left(X,{ }^{*}\right) \rightarrow(Y, *)$ be a pointed homotopy equivalence of $L C^{\circ}$ M-L-F compacta. If $\bar{X}$ and $\bar{Y}$ are path connected then the induced map $\bar{f}:\left(\bar{X},{ }^{*}\right) \rightarrow\left(\bar{Y},{ }^{*}\right)$ is a pointed proper homotopy equivalence.

Proof. By Theorem 5.9 and the homotopy lifting property for fibrations with unique path lifting $\bar{f}$ is a pointed homotopy equivalence. We prove $\bar{f}$ is proper and a similar argument shows that the compositions of $\bar{f}$ with its pointed homotopy inverse are pointedly proper homotopic to the appropriate identity map. Let $U_{1}, U_{2}, \cdots, U_{n}$ be evenly covered compact neighborhoods covering $X_{1}$, and let $\widetilde{U}_{i}$ be a homeomorphic copy of $U_{i}$ in $p_{1}^{-1}\left(U_{i}\right) .\left\{x \cdot \widetilde{U}_{i} \mid x \in F_{1}\right.$ and $i \epsilon$ $\{1,2, \cdots, n\}\}$ is a locally finite cover of $\widetilde{X}_{1}$. Recall $\tilde{\alpha}_{1}:\left(\bar{X},{ }^{*}\right) \rightarrow\left(\widetilde{X}_{1},{ }^{*}\right)$, is proper. Choose $w_{x} \in \widetilde{\alpha}_{1}^{-1}(x)$ for each $x \in F_{1}$ and let $V_{i}=\widetilde{\alpha}_{1}^{-1}\left(\widetilde{U}_{i}\right)$, $\left\{w_{x} \mid x \in F_{1}\right\}$ is a discrete subset of $F$ and $\left\{w_{x} \cdot V_{i} \mid x \in F_{1}\right.$ and $i \epsilon$ $\{1,2, \cdots, n\}\}$ is a locally finite cover of $\bar{X}$ by compact neighborhoods. Since $\bar{f}$ is a pointed homotopy equivalence, $\bar{f}$ maps $p^{-1}(*)$ homeomorphically onto $q^{-1}\left({ }^{*}\right)$. The rest of the proof is the same as that of Proposition 5.2 with $\tilde{e}_{i}$ replaced by $V_{i}$ and $g_{i}$ replaced by $w_{x_{i}}$ where $x_{i} \in F_{1}$.

\section{REFERENCES}

1. M. Atiyah and G. B. Segal, Equivariant K-theory and completion, J. Differential Geometry, 3 (1969), 1-18.

2. N. Bourbaki, General Topology, Addison-Wesely, Reading, Mass., 1966.

3. M. Cohen, A Course in Simple-Homotopy Theory, Graduate texts in Math. 10, Springer-Verlag, Berlin and New York, 1973.

4. J. Dugundi, Topology, Allyn and Bacon, Boston, 1966. 
5. J. Dydak and J. Segal, Shape Theory, Lecture Notes in Math., vol. 688, SpringerVerlag, Berlin and New York, 1978.

6. D. A. Edwards and R. Geoghegan, Compacta weak shape equivalent to ANR's, Fund. Math., 90 (1976), 115-124.

7. S. Ferry, A stable converse to the Vietoris-Smale theorem with applications to shape theory, (pre-print).

8. H. Freudenthal, Über die Enden topologischer Raiime und Gruppen, Math. Zeir., 33 (1931), 692-713.

9. A. Grothendieck, Technique de descente et theoremes d'existence en geometrie alge. brique II, Seminaire Bourbaki, 12-ieme annee, (1959-60) Exp. 195.

10. H. Hopf, Enden offener Räume und unendliche diskontinuierliche Grouppen, Comm. Math. Helv., 16 (1943), 81-100.

11. J. Krasinciewicz, Local connectedness and pointed 1-movability, Bulletin de L'Academie Polonaise des Sciences, XXV (1977).

12. R. Lyndon and P. Schupp, Combinatorial Group Theory, Ergebnisse, der Mathematik und ihrer Grenzgebiete Vol.89, Springer-Verlag, Berlin and New York, 1972.

13. E. H. Spanier, Algebraic Topology, McGraw-Hill, New York, 1966.

14. J. Stallings, Group theory and three dimensional manifolds, Yale Math. Monographs

4, Yale University Press, New Haven, 1972.

Received May 4, 1979 and in revised form September 19, 1979.

State University of New York at Binghamton

Binghamton, NY 13901 


\section{PACIFIC JOURNAL OF MATHEMATICS}

\section{EDITORS}

DONALD BABBITT (Managing Editor)

University of California

Los Angeles, CA 90024

Hugo RossI

University of Utah

Salt Lake City, UT 84112

C. C. MOORE and ANDREW OGG

University of California

Berkeley, CA 94720

\section{J. DugunduI}

Department of Mathematics

University of Southern California

Los Angeles, CA 90007

R. Finn and J. Milgram

Stanford University

Stanford, CA 94305

\section{ASSOCIATE EDITORS}
E. F. BeCKenBACH
B. H. NeumanN
F. WOLF
K. YosHIDA

\section{SUPPORTING INSTITUTIONS}

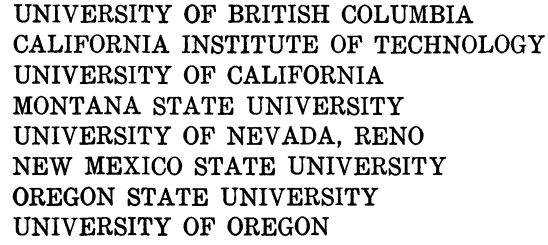

UNIVERSITY OF BRITISH COLUMBIA CALIFORNIA INSTITUTE OF TECHNOLOGY UNIVERSITY OF CALIFORNIA

MONTANA STATE UNIVERSITY

UNIVERSITY OF NEVADA, RENO

NEW MEXICO STATE UNIVERSITY

OREGON STATE UNIVERSITY UNIVERSITY OF OREGON

\author{
UNIVERSITY OF SOUTHERN CALIFORNIA \\ STANFORD UNIVERSITY \\ UNIVERSITY OF HAWAII \\ UNIVERSITY OF TOKYO \\ UNIVERSITY OF UTAH \\ WASHINGTON STATE UNIVERSITY \\ UNIVERSITY OF WASHINGTON
}

The Supporting Institutions listed above contribute to the cost of publication of this Journal, but they are not owners or publishers and have no responsibility for its content or policies.

Mathematical papers intended for publication in the Pacific Journal of Mathematics should be in typed form or offset-reproduced, (not dittoed), double spaced with large margins. Please do not use built up fractions in the text of the manuscript. However, you may use them in the displayed equations. Underline Greek letters in red, German in green, and script in blue. The first paragraph or two must be capable of being used separately as a synopsis of the entire paper. Please propose a heading for the odd numbered pages of less than 35 characters. Manuscripts, in triplicate, may be sent to any one of the editors. Please classify according to the scheme of Math. Reviews, Index to Vol. 39. Supply name and address of author to whom proofs should be sent. All other communications should be addressed to the managing editor, or Elaine Barth, University of California, Los Angeles, California, 90024.

50 reprints to each author are provided free for each article, only if page charges have been substantially paid. Additional copies may be obtained at cost in multiples of 50 .

The Pacific Journal of Mathematics is issued monthly as of January 1966. Regular subscription rate: $\$ 84.00$ a year (6 Vols., 12 issues). Special rato: $\$ 42.00$ a year to individual members of supporting institutions.

Subscriptions, orders for numbers issued in the last three calendar years, and changes of address shoud be sent to Pacific Journal of Mathematics, P.O. Box 969, Carmel Valley, CA 93924, U.S.A Old back numbers obtainable from Kraus Periodicals Co., Route 100, Millwood, NY 10546.

PUBLISHED BY PACIFIC JOURNAL OF MATHEMATICS, A NON-PROFIT CORPORATION

Printed at Kokusai Bunken Insatsusha (International Academic Printing Co., Ltd.). 8-8, 3-chome, Takadanobaba, Shinjuku-ku, Tokyo 160, Japan.

Copyright (C) 1980 by Pacific Jounal of Mathematics Manufactured and first issued in Japan 


\section{Pacific Journal of Mathematics \\ Vol. 90, No. $2 \quad$ October, 1980}

Frank Hayne Beatrous, Jr., Hölder estimates for the $\bar{\partial}$ equation with a support condition ..................................... 249

Charles L. Belna, Michael Jon Evans and Paul Humke, Planar continua

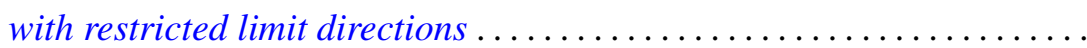

Leon Brown and Takashi Ito, Classes of Banach spaces with unique isometric preduals................................. 261

V. K. Deshpande, Completions of Noetherian hereditary prime rings ..... . 285

Deepak Dhar, Asymptotic enumeration of partially ordered sets . . . . . . . 299

Zeev Ditzian, On interpolation of $L_{p}[a, b]$ and weighted Sobolev

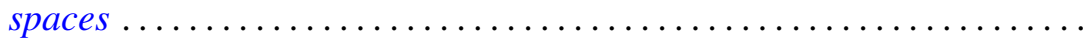

Andrew George Earnest, Congruence conditions on integers represented by

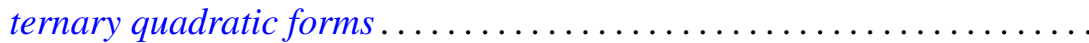

Melvin Faierman, Bounds for the eigenfunctions of a two-parameter system of ordinary differential equations of the second order ..............

Hector O. Fattorini, Vector-valued distributions having a smooth

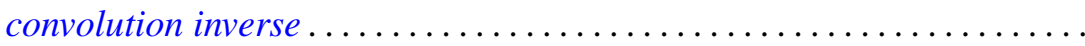

Howard D. Fegan, The spectrum of the Laplacian on forms over a Lie

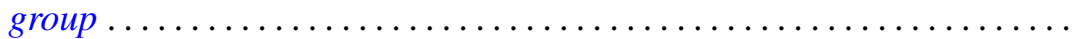

Gerald Leonard Gordon, On the degeneracy of a spectral sequence associated to normal crossings..

S. Madhavan, On bisimple weakly inverse semigroups ... 397

Françoise Mathot, On the decomposition of states of some

Roger McCann, Embedding asymptotically stable dynamical systems into radial flows in $l_{2}$

Michael L. Mihalik, Ends of fundamental groups in shape and proper homotopy...............................

Samuel Murray Rankin, III, Boundary value problems for partial functional differential equations .

Randy Tuler, Arithmetic sums that determine linear characters on $\Gamma(N)$

Jeffrey D. Vaaler, On linear forms and Diophantine approximation ..

G. P. Wene, Alternative rings whose symmetric elements are nilpotent or a right multiple is a symmetric idempotent. . 